\title{
Calculating the dimension of the universal embedding of the symplectic dual polar space using languages
}

\author{
Carlos Segovia* \\ Instituto de Matemáticas UNAM \\ Oaxaca, Mexico \\ csegovia@matem.unam.mx
}

\author{
Monika Winklmeier \\ Department of Mathematics \\ University of the Andes \\ Bogotá, Colombia \\ mwinklme@uniandes. edu. co
}

Submitted: Aug 2, 2020; Accepted: Nov 11, 2020; Published: Dec 11, 2020

(C) The authors. Released under the CC BY-ND license (International 4.0).

\begin{abstract}
The main result of this paper is the construction of a bijection of the set of words in so-called standard order of length $n$ formed by four different letters and the set $\mathcal{N}^{n}$ of all subspaces of a fixed $n$-dimensional maximal isotropic subspace of the $2 n$-dimensional symplectic space $V$ over $\mathbb{F}_{2}$ which are not maximal in a certain sense. Since the number of different words in standard order is known, this gives an alternative proof for the formula of the dimension of the universal embedding of a symplectic dual polar space $\mathcal{G}_{n}$. Along the way, we give formulas for the number of all $n$ - and $(n-1)$-dimensional totally isotropic subspaces of $V$.
\end{abstract}

Mathematics Subject Classifications: 05B25, 68R15

\section{Introduction}

Configurations of points and lines are of significant importance since they occur for instance as designs in combinatorics, geometry and algebra. These structures have been extensively documented in [Lev29, Grü09, PS13], and historically, projective geometry has provided important examples like the Fano plane [Dem68]. The configurations induced by a projective geometry are completely characterized by a set of axioms for its points and lines, and analogously we can find the configurations induced by the (dual) polar spaces. The axiomatic formulation of a polar space was given in [BS74], while the axioms for a dual polar space were developed in [Cam82]. An important example of a polar space is

${ }^{*}$ C. Segovia is supported by catedras CONACYT and Proyecto CONACYT ciencias básicas 2016, No. 284621 . 


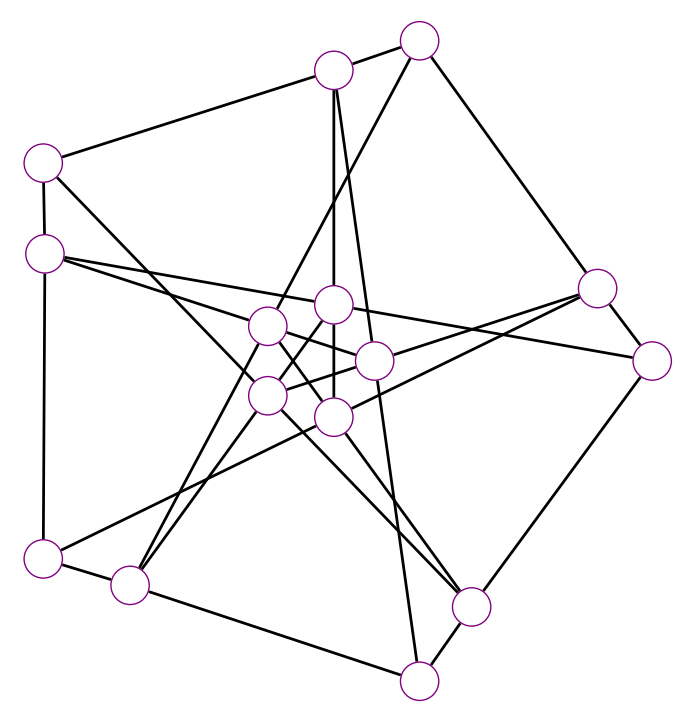

Figure 1: The Cremona-Richmond configuration.

the set of all totally isotropic subspaces of a given symplectic space whereas the set of all maximal isotropic subspaces form a dual polar space.

In this article we consider a symplectic space $V$ of dimension $2 n$. We denote by $\mathcal{P}_{n}$ the set of all maximal totally isotropic subspaces of $V$ and by $\mathcal{L}_{n}$ the set of all totally isotropic subspaces of dimension $n-1$. They form a configuration of points and lines $\mathcal{G}_{n}=\left(\mathcal{P}_{n}, \mathcal{L}_{n}\right)$ called the symplectic dual polar space, where the incidence relation is given by inclusion of the subspaces. In the case when $V$ is a $\mathbb{F}_{2}$-vector space, this structure is completely understood and there is a vast literature on this matter [BCN89, Bru06, BC13]. The case $n=2$ is of great importance because it gives the self-dual configuration called Cremona-Richmond configuration [Cre77, Ric00] whose exciting history can be found in [Bak10a, Bak10b]. In Figure 1 we show the Cremona-Richmond configuration, which has fifteen points and fifteen lines such that every point is contained in exactly three lines and every line contains exactly three different points. Starting from the symplectic dual polar space $\mathcal{G}_{n}$, we construct its universal embedding $U\left(\mathcal{G}_{n}\right):=\mathbb{F}_{2}\left(\mathcal{P}_{n}\right) / \eta\left(\mathbb{F}_{2}\left(\mathcal{L}_{n}\right)\right)$, where $\eta$ : $\mathbb{F}_{2}\left(\mathcal{L}_{n}\right) \rightarrow \mathbb{F}_{2}\left(\mathcal{P}_{n}\right)$ sends every line to the sum of its three elements. Brouwer conjectured that the value of $\operatorname{dim}\left(U\left(\mathcal{G}_{n}\right)\right)$ is given by the sequence $\left(x_{n}\right)_{n \in \mathbb{N}}=(2,5,15,51,187, \ldots)$ with $x_{n}=\left(2^{n}+1\right)\left(2^{n-1}+1\right) / 3$ which is the sequence A007581 in [Slo]. This conjecture was proved by P. Li in [Li01] and independently by A. Blokhuis and A. E. Brouwer in [BB03]. In this paper we are mainly concerned with the procedure employed by $\mathrm{P}$. $\mathrm{Li}$ in [Li01] where he considers sets $\mathcal{N}^{n}$ of subspaces of a fixed $n$-dimensional maximal isotropic subspace of a $2 n$-dimensional symplectic space $V$ over $\mathbb{F}_{2}$. These subsets $\mathcal{N}^{n}$ are not maximal in a sense to be made precise in Section 3. Every set $\mathcal{N}^{n}$ is subdivided into a disjoint union of families which are constructed inductively. In our work we construct a bijection between 
the set $\mathcal{N}^{n}$ and a set of words of length $n$ in so-called standard order, formed by four different letters. Moreover, this bijection respects the inductive construction thus allowing us to construct every element of $\mathcal{N}^{n}$ in a very simple way. As a consequence, our procedure gives an alternative proof of the formula for the dimension of the universal embedding $U\left(\mathcal{G}_{n}\right)$ since the number of words can be easily counted. This construction establishes a relationship between the first and the second of the many different interpretations of the sequence $\left(x_{n}\right)_{n \in \mathbb{N}}=(2,5,15,51,187, \ldots)$ (the sequences A007581 and A124303 in [Slo]) in the following list:

1. The dimension of the universal embedding of the symplectic dual polar space [BB03, Li01].

2. The density of a language with four letters [MR05, SW15].

3. The number of isomorphism classes of regular fourfold coverings of a graph $L$ with Betti number $n=\beta(L)$ and with voltage group $\mathbb{F}_{2} \times \mathbb{F}_{2}$ [HK93].

4. The number of non-equivalent states of a Hanoi graph associated to the Hanoi tower with $n$ discs and four pegs [HKMP13].

5. The dimension of a certain centralizer algebra associated to a group of order 96 [KO16].

6. The dimension of the space of symmetric polynomials in 4 noncommuting variables [BRRZ08, RS06].

7. An invariant of the group $\mathbb{Z}_{2}^{n}$ of cobordism type, see [Seg19, CS18].

Actually, all this is part of a more general setting with an arbitrary prime number $p$. In [SW15] we considered a language with $p^{2}$ letters as a quotient of $\left(\mathbb{Z}_{p} \times \mathbb{Z}_{p}\right)^{n}$ by the special linear group $S L(2, \mathbb{Z})$. In the case of a dual polar space, we consider the totally isotropic subspaces of an $\mathbb{F}_{p}$-vector space $V$, where we get configurations with points $\mathcal{P}_{n}$ and lines $\mathcal{L}_{n}$ satisfying

$$
\left|\mathcal{P}_{n}(p)\right|=\prod_{k=1}^{n}\left(p^{k}+1\right) \text { and }\left|\mathcal{L}_{n}(p)\right|=\frac{\left(p^{n}-1\right)}{p^{2}-1} \prod_{k=1}^{n}\left(p^{k}+1\right),
$$

where every line has $p+1$ points and every point is contained in $\frac{p^{n}-1}{p-1}$ lines. For instance, for $n=2$ this produces a sequence of self-dual configurations $\left((p+1)\left(p^{2}+1\right)\right)_{p+1}$ for prime numbers $p$, i.e., $15_{2+1}, 40_{3+1}, 156_{5+1}, 400_{7+1}, \ldots$ which we will call the $p$-CremonaRichmond configurations. The sequence $15,40,156,400,1464, \ldots$ appears as the sequence A131991 in [Slo].

It is not a coincidence that there are many different but equivalent approaches to the sequence $\left(x_{n}\right)_{n \in \mathbb{N}}=(2,5,15,51,187, \ldots)$ and the mathematics involved is of great interest. In terms of a language with four letters, we have a correspondence with ordered set partitions producing some type of quasi-Young diagrams which gives the dimension of 
the space of symmetric polynomials in four noncommuting variables [RS06]. The results of the present work produce a bijection between a language and a base for the symplectic Grassmannian of a dual polar space. In fact, this resembles the case for partitions when we count the number of irreducible representations of the symmetric groups and in a certain way, we have the number of $n$-cells for the Grassmannian [MS74].

The paper is organized as follows. In Section 2 we discuss the set $W^{n}$ of words of length $n$ in so-called standard order formed by four letters and we give a procedure for the construction of all the words $W^{n+1}$ from the ones in $W^{n}$. We obtain two proofs for the formula for $\left|W^{n}\right|$ (proof of formula (1) on page 7 and Remark 6). In Sections 3 we outline several facts on isotropic subspaces of symplectic $\mathbb{F}_{2}$-vector spaces and the symplectic dual polar space. Additionally, we review Li's proof for the formula of the dimension of the universal embedding of the symplectic polar space $\operatorname{Sp}_{2 n}(2)$ which allows us to construct the bijection between the words $W^{n}$ and Li's vector spaces $\mathcal{N}^{n+1}$ in Section 4 . This gives a new proof for the formula for the dimension of the universal embedding of the symplectic dual polar space in Theorem 18. In Appendix A we present the decomposition of the collinearity graph $\Gamma$ for $n=2$ and $n=3$ in its subgraphs $\Gamma_{k}$. In Appendix B we show the construction of $W^{n+1}$ from $W^{n}$ for $n=1,2,3,4$. Finally, in Appendix C, we present a classification of words in $W^{n}$ according to the eight cases specified in Section 2.

\section{Languages}

Let us consider a language with the four letters $0,1,2,3$. For $n \in \mathbb{N}$ we define $\widetilde{W}^{n}:=$ $\left\{a_{1} \ldots a_{n}: a_{j}=0,1,2,3\right\}$ to be the set of all possible words of length $n$ formed by the letters $0,1,2,3$. In this article we will be mainly concerned with the subset $W^{n}$ of words in the so-called standard order [AS16, MR05]. The set $W^{n}$ consists of the words $a_{1} a_{2} \ldots a_{n} \in \widetilde{W}^{n}$ such that there exist $1 \leqslant j<k$ with:

(R1) $a_{i}=0$ for $i<j$,

(R2) $a_{j}=1$,

(R3) $a_{i} \in\{0,1\}$ for $j<i<k$,

(R4) $a_{k}=2$ if $k \leqslant n$,

(R5) $a_{i} \in\{0,1,2,3\}$ for $i>k$.

Note that (R5) applies only if $k<n$. For a word $a=a_{1} a_{2} \ldots a_{n}$ the rules above can be written compactly as

$$
0 \leqslant a_{i} \leqslant \max _{j<i}\left\{a_{j}\right\}+1, \quad 1 \leqslant i \leqslant n .
$$

Note that our set $W^{n}$ is the special case $W_{2}^{n}$ of the more general sets $W_{p}^{n}$ defined in [SW15] for arbitrary prime numbers $p$.

Definition 1. The cardinality of $W^{n}$ is called the density of the language $W^{n}$. We use the notation $g_{W}(n):=\left|W^{n}\right|$. 
Example 2. We have $W^{1}=\{0\}$, consisting of 1 word, $W^{2}=\{00,01\}$, consisting of 2 words, $W^{3}=\{000,001,010,011,012\}$, consisting of 5 words. For $n=4$ there are 15 words and the elements of $W^{4}$ are

$\begin{array}{lllll}0000 & 0001 & 0010 & 0011 & 0012 \\ 0100 & 0101 & 0102 & 0110 & 0111 \\ 0112 & 0120 & 0121 & 0122 & 0123 .\end{array}$

The following theorem was already shown in [MR05] and [SW15].

Theorem 3. For $n \in \mathbb{N}_{0}$ the density of the language $W^{n}$ is

$$
\left|W^{n}\right|=g_{W}(n)=\frac{\left(2^{n-1}+1\right)\left(2^{n-2}+1\right)}{3} .
$$

In the present work, we want to provide a different point of view, motivated by the work of $\mathrm{Li}$ [Li01], and we will give an alternative proof of Theorem 3 after Proposition 4. In what follows we study some facts which are fundamental for the proof of this theorem.

Let $n \geqslant 2$. We will show how all words in $W^{n+1}$ can be constructed from the words in $W^{n}$.

- Case 1. Take an arbitrary word in $W^{n}$ and attach 0 at the end. This gives a valid word in $W^{n+1}$. The number of all such words is $g_{W}(n)$.

- Case 2. Take an arbitrary word in $W^{n}$ and attach 1 at the end. This gives a valid word in $W^{n+1}$ and it is not contained in the words obtained in case 1 . The number of all such words is $g_{W}(n)$.

For Cases 3, 4, 5 we take an arbitrary word $a=a_{1} a_{2} \ldots a_{n}$ in $W^{n}$ which ends in 2 or 3. Note that this implies $1 \in\left\{a_{1}, \ldots, a_{n-1}\right\}$ and that therefore $a=a_{1} a_{2} \ldots a_{n-1} \ell a_{n}$ is a valid word in $W^{n+1}$ for $\ell=0,1,2$.

- Case 3. Insert the letter 0 before $a_{n}$. Then we obtain the valid word $\widetilde{a}=$ $a_{1} a_{2} \ldots a_{n-1} 0 a_{n} \in W^{n+1}$. Clearly this word is not contained in the words constructed so far.

- Case 4. Insert the letter 1 before $a_{n}$. Then we obtain the valid word $\widetilde{a}=$ $a_{1} a_{2} \ldots a_{n-1} 1 a_{n} \in W^{n+1}$. Clearly this word is not contained in the words constructed so far.

- Case 5. Insert the letter 2 before $a_{n}$. Then we obtain the valid word $\widetilde{a}=$ $a_{1} a_{2} \ldots a_{n-1} 2 a_{n} \in W^{n+1}$. Clearly this word is not contained in the words constructed so far.

The number of words in each of the Cases $3,4,5$ is

\#(words of length $n$ ending in 2 or 3$)=g_{W}(n)-\#($ words of length $n$ ending in 0 or 1$)$

$$
=g_{W}(n)-2 g_{W}(n-1),
$$

since \#(words of length $n$ ending in 0$)=\#($ words of length $n$ ending in 1$)=g_{W}(n-1)$ as in Case 1 and Case 2. 
- Case 6. Let $a=a_{1} a_{2} \ldots a_{n}$ in $W^{n}$ which ends in 2 or 3 and such that $2 \in$ $\left\{a_{1}, \ldots, a_{n-1}\right\}$.

Insert the letter 3 before $a_{n}$. We obtain the valid word $\widetilde{a}=a_{1} a_{2} \ldots a_{n-1} 3 a_{n} \in W^{n+1}$. Clearly this word is not contained in the words constructed so far.

- Case 7. Let $a=a_{1} a_{2} \ldots a_{n}$ in $W^{n}$ which ends in 2 or 3 and such that $2 \notin$ $\left\{a_{1}, \ldots, a_{n-1}\right\}$.

This implies that $a_{n}=2$ and $a_{j} \in\{0,1\}$ for $1 \leqslant j \leqslant n-1$. Attach 3 to obtain the new word $\widetilde{a}=a_{1} a_{2} \ldots a_{n-1} 23 \in W^{n+1}$. Clearly this word is not contained in the words constructed so far. The number of all such words is equal to the number of strings of length $n-1$ consisting only of 0 and 1 , with exception of the zero string. So the number of the words in this case is $2^{n-2}-1$.

The total number of words in the Cases 6 and 7 together is \#(words of length $n$ ending in 2 or 3$)=g_{W}(n)-\#($ words of length $n$ ending in 0 or 1$)$

$$
=g_{W}(n)-2 g_{W}(n-1) \text {. }
$$

- Case 8. Let $\widetilde{a}=0 \ldots 012 \in W^{n+1}$. Clearly this word is not contained in the words constructed so far.

We say that a word $\widetilde{a} \in W^{n+1}$ is in Case $k$ for $k=1, \ldots, 8$, if it is constructed from a word $a \in W^{n}$ as described in Case $k$.

Proposition 4. Let $n \geqslant 2$. Then each word in $W^{n+1}$ is constructed as in exactly one of the Cases $1-8$ above.

Proof. Let $\widetilde{a}=a_{1} a_{2} \ldots a_{n} a_{n+1} \in W^{n+1}$.

- Suppose that $a_{n+1} \in\{0,1\}$. Then clearly $\widetilde{a}$ is constructed either as in Case 1 or in Case 2.

- Suppose that $a_{n+1} \in\{2,3\}$. Note that this implies $1 \in\left\{a_{1}, \ldots, a_{n}\right\}$.

- If $a_{n}=0$, we can erase it and obtain the valid word $a=a_{1} \ldots a_{n-1} a_{n+1} \in W^{n}$. If we now apply the procedure of Case 3 , we recover $\widetilde{a}$.

- If $a_{n}=1$ and $a=a_{1} \ldots a_{n-1} a_{n+1}$ is a valid word in $W^{n}$, then we can apply the procedure of Case 4 and we obtain again $\widetilde{a}$. If $a=a_{1} \ldots a_{n-1} a_{n+1}$ is not a valid word in $W^{n}$, then necessarily $\widetilde{a}=0 \ldots 012$ and we have the word of Case 8 .

- If $a_{n}=2$ and $a=a_{1} \ldots a_{n-1} a_{n+1}$ is a valid word in $W^{n}$, then we can apply the procedure of Case 5 and we obtain again $\widetilde{a}$. If $a=a_{1} \ldots a_{n-1} a_{n+1}$ is not a valid word in $W^{n}$, then necessarily $a_{n+1}=3$ and $2 \notin\left\{a_{1}, \ldots, a_{n-1}\right\}$. Then we can apply the procedure of Case 7 to the word $a^{\prime}=a_{1} \ldots a_{n-1} a_{n}$ and we recover $\widetilde{a}$. 
- If $a_{n}=3$ then necessarily $a=a_{1} \ldots a_{n-1} a_{n+1}$ is a valid word in $W^{n}$ and we can apply the procedure of Case 6 to recover $\widetilde{a}$.

In Appendix $\mathrm{C}$ we give a classification of the words of $W^{n}$ according to the cases described above and in Appendix B we show explicitly how $W^{n+1}$ is constructed from $W^{n}$ for $n=2,3,4$. Proposition 4 allows us to prove the formula (1) as follows.

Proof of Formula (1). By Proposition 4 we know that, for $n \geqslant 2$,

$$
\begin{aligned}
\left|W^{n+1}\right|=g_{W}(n+1) & =2 g_{W}(n)+4\left[g_{W}(n)-2 g_{W}(n-1)\right]+1 \\
& =6 g_{W}(n)-8 g_{W}(n-1)+1
\end{aligned}
$$

From Example 2 we obtain that $\left|W^{1}\right|=1,\left|W^{2}\right|=2$, hence formula (1) is satisfied for $n=1,2$. Now suppose that the formula holds for all $j \leqslant n$. Then

$$
\begin{aligned}
g_{W}(n+1) & =6 g_{W}(n)-8 g_{W}(n-1)+1 \\
& =6 \frac{\left(2^{n-1}+1\right)\left(2^{n-2}+1\right)}{3}-8 \frac{\left(2^{n-2}+1\right)\left(2^{n-3}+1\right)}{3}+1 \\
& =\frac{\left(2^{n-2}+1\right)}{3}\left[6\left(2^{n-1}+1\right)-8\left(2^{n-3}+1\right)\right]+1 \\
& =\frac{2\left(2^{n-2}+1\right)\left(2^{n}-1\right)+3}{3} \\
& =\frac{2^{2 n-1}+2^{n+1}-2^{n-1}+1}{3}=\frac{2^{2 n-1}+2^{n}+2^{n-1}+1}{3} \\
& =\frac{\left(2^{n}+1\right)\left(2^{n-1}+1\right)}{3} .
\end{aligned}
$$

Remark 5. An alternative proof of Proposition 4 makes use of the formula (1) for $\left|g_{W}(n)\right|$ which was already proved in [MR05] and [SW15]. Then it is sufficient to prove that the number of words obtained by the Cases 1 to 8 is equal to $\left|W^{n+1}\right|$ because we already know that all cases are disjoint and that every word constructed in these cases belongs to $W^{n+1}$. That is, we have to show that

$$
g_{W}(n+1)=2 g_{W}(n)+6\left[g_{W}(n)-g_{W}(n-1)\right]+1 .
$$

This is a straightforward calculation.

Proposition 4 gives yet another way to calculate $\left|W^{n}\right|$ as the next remark shows.

Remark 6. We showed that with the rules in Cases 1 to 8 , each word in $W^{n}$ which ends in 0 or 1 gives rise to exactly two words in $W^{n+1}$ (Cases 1 and 2). They again end in 0 or 1 . Each word in $W^{n}$ which ends in 2 or 3 gives rise to exactly six words in $W^{n+1}$, two of which end in 0 or 1, and four of them end again in 2 or 3 (Cases 3, 4, 5 and either 6 or 7). In addition we have the word from Case 8. 
Let $g_{W}(n)=\left|W^{n}\right|$ and

$$
\begin{aligned}
& s(n)=\text { number of words in } W^{n} \text { which end in } 0 \text { or } 1, \\
& t(n)=\text { number of words in } W^{n} \text { which end in } 2 \text { or } 3 .
\end{aligned}
$$

Then we obtain $t(1)=t(2)=0, t(3)=1, s(1)=1, s(2)=2, s(3)=4$ and for $n \geqslant 2$

$$
g_{W}(n+1)=2 s(n)+6 t(n)+1, \quad s(n+1)=2 s(n)+2 t(n), \quad t(n+1)=4 t(n)+1 .
$$

Iterating the formula for $t(n)$, we find $t(n)=\frac{4^{n-2}-1}{3}$. For $s(n)$ we find

$$
\begin{aligned}
s(n) & =2^{n-3} s(3)+\sum_{j=1}^{n-3} 2^{j} t(n-j)=2^{n-1}+\frac{1}{3} \sum_{j=1}^{n-3} 2^{j}\left(2^{2 n-4-2 j}-1\right) \\
& =2^{n-1}+\frac{2}{3}\left(\sum_{j=0}^{n-4} 2^{2 n-6-j}-\sum_{j=0}^{n-4} 2^{j}\right) \\
& =2^{n-1}+\frac{2}{3}\left(2^{n-2}-1\right) \sum_{j=0}^{n-4} 2^{j}=2^{n-1}+\frac{2}{3}\left(2^{n-2}-1\right)\left(2^{n-3}-1\right) \\
& =\frac{1}{3}\left(3 \cdot 2^{n-1}+2^{2 n-4}-2^{n-1}-2^{n-2}+2\right)=\frac{1}{3}\left(2^{2 n-4}+2^{n-1}+2^{n-2}+2\right) .
\end{aligned}
$$

So we find again formula (1) for $g_{W}(n)$ :

$$
\begin{aligned}
g_{W}(n) & =s(n)+t(n)=\frac{1}{3}\left[2^{2 n-4}+2^{n-1}+2^{n-2}+2+4^{n-2}-1\right]=\frac{1}{3}\left[2^{2 n-3}+2^{n-1}+2^{n-2}+1\right] \\
& =\frac{\left(2^{n-1}+1\right)\left(2^{n-2}+1\right)}{3} .
\end{aligned}
$$

Definition 7. Let us introduce some more notation. We define the following subsets of $W^{n}$ :

$W_{0}^{n}:=\left\{a_{1} \ldots a_{n-1} 0: a_{j}=0,1,2,3\right\}=$ all words ending in 0,

$W_{1}^{n}:=\left\{a_{1} \ldots a_{n-1} 1: a_{j}=0,1,2,3\right\}=$ all words ending in 1 ,

$S_{0}^{n}:=\left\{a_{1} \ldots a_{n-2} 0 b_{n}: a_{j}=0,1,2,3, b_{n}=2,3\right\}=$ all words ending in 2 or 3 with $a_{n-1}=0$,

$S_{1}^{n}:=\left\{a_{1} \ldots a_{n-2} 1 b_{n}: a_{j}=0,1,2,3, b_{n}=2,3\right\}=$ all words ending in 2 or 3 with $a_{n-1}=1$,

$S_{2}^{n}:=\left\{a_{1} \ldots a_{n-2} 2 b_{n}: a_{j}=0,1,2,3, b_{n}=2,3\right\}=$ all words ending in 2 or 3 with $a_{n-1}=2$,

$S_{3}^{n}:=\left\{a_{1} \ldots a_{n-2} 3 b_{n}: a_{j}=0,1,2,3, b_{n}=2,3\right\}=$ all words ending in 2 or 3 with $a_{n-1}=3$,

$S^{n}:=S_{0}^{n} \cup S_{1}^{n} \cup S_{2}^{n} \cup S_{3}^{n}$,

$C_{1}^{n}:=\left\{a_{1} \ldots a_{n-2} 23: a_{j}=0,1\right\}$,

$C_{2}^{n}:=\left\{a_{1} \ldots a_{n-1} 2: a_{j}=0,1\right\}=$ all words with $a_{n}=2$ and no other 2 ,

$C_{3}^{n}:=\{0 \ldots 012\}$. 
Observe that $C_{1}^{n} \subset S_{2}^{n}, C_{3}^{n} \subset S_{1}^{n}$ and that

$$
W^{n+1}=W_{0}^{n+1} \sqcup W_{1}^{n+1} \sqcup S_{0}^{n+1} \sqcup S_{1}^{n+1} \sqcup S_{2}^{n+1} \sqcup S_{3}^{n+1}
$$

where $\sqcup$ denotes the disjoint union. Now we define the insert operators for $k=1, \ldots, n+1$ and $\ell=0,1,2,3$ as follows:

$$
A_{k, \ell}^{n}: W^{n} \rightarrow \widetilde{W}^{n+1}, \quad A_{k, \ell}^{n}\left(a_{1} \ldots a_{n}\right)=a_{1} \ldots a_{k-1} \ell a_{k} \ldots a_{n} .
$$

For $n \in \mathbb{N}$ and $1 \leqslant j \leqslant n$ we define the erase operators

$$
E_{j}^{n}: W^{n} \rightarrow \widetilde{W}^{n-1}, \quad E_{j}^{n}\left(a_{1} \ldots a_{n}\right)=a_{1} \ldots a_{j-1} a_{j+1} \ldots a_{n} .
$$

It should be observed that for $a \in W^{n}$ and $j \in\{1,2, \ldots, n\}$, the word $E_{j}^{n}(a)$ is not necessarily a word in $W^{n-1}$.

With this new notation, the results of this section so far can be summarized as follows. Theorem 8 is essentially Proposition 4 with the constructions of words expressed by maps which will be useful later in Theorem 9 and Theorem 17.

Theorem 8. Let $n \in \mathbb{N}$. Then the following maps are bijections:

$$
\begin{aligned}
& A_{n, 0}^{n}: \quad S^{n} \rightarrow S_{0}^{n+1}, \\
& A_{n, 1}^{n}: \quad S^{n} \rightarrow S_{1}^{n+1} \backslash C_{3}^{n+1}, \quad(\text { Case 4) } \\
& A_{n+1,0}^{n}: W^{n} \rightarrow W_{0}^{n+1}, \quad(\text { Case } 1) \\
& A_{n+1,1}^{n}: W^{n} \rightarrow W_{1}^{n+1}, \quad(\text { Case } 2) \\
& A_{n, 2}^{n}: \quad S^{n} \rightarrow S_{2}^{n+1} \backslash C_{1}^{n+1}, \\
& A_{n, 3}^{n}: S^{n} \backslash C_{2}^{n} \rightarrow S_{3}^{n+1}, \\
& A_{n+1,3}^{n}: \quad C_{2}^{n} \rightarrow C_{1}^{n+1}
\end{aligned}
$$

and $W^{n+1}=W_{0}^{n+1} \sqcup W_{1}^{n+1} \sqcup S_{0}^{n+1} \sqcup\left(S_{1}^{n+1} \backslash C_{3}^{n+1}\right) \sqcup\left(S_{2}^{n+1} \backslash C_{1}^{n+1}\right) \sqcup S_{3}^{n+1} \sqcup C_{1}^{n+1} \sqcup C_{3}^{n+1}$ is the disjoint union of the images of the maps above and $C_{3}^{n+1}$.

The inverses of the maps above are

$$
\begin{array}{ll} 
& E_{n}^{n+1}: \quad S_{0}^{n+1} \rightarrow S^{n}, \\
E_{n+1}^{n+1}: W_{0}^{n+1} \rightarrow W^{n}, & E_{n}^{n+1}: S_{1}^{n+1} \backslash C_{3}^{n+1} \rightarrow S^{n}, \\
E_{n+1}^{n+1}: W_{1}^{n+1} \rightarrow W^{n}, & E_{n}^{n+1}: S_{2}^{n+1} \backslash C_{1}^{n+1} \rightarrow S^{n}, \\
& E_{n}^{n+1}: \quad S_{3}^{n+1} \rightarrow S^{n} \backslash C_{2}^{n}, \\
& E_{n+1}^{n+1}: \quad C_{1}^{n+1} \rightarrow C_{2}^{n} .
\end{array}
$$

Moreover,

$$
\begin{aligned}
\left|W_{0}^{n+1}\right|=\left|W_{1}^{n+1}\right| & =g_{W}(n), \\
\left|S_{0}^{n+1}\right|=\left|S_{1}^{n+1} \backslash C_{3}^{n+1}\right|=\left|S_{2}^{n+1} \backslash C_{1}^{n+1}\right| & =g_{W}(n)-2 g_{W}(n-1), \\
\left|S_{3}^{n+1}\right|+\left|C_{1}^{n+1}\right| & =g_{W}(n)-2 g_{W}(n-1) .
\end{aligned}
$$

Theorem 9. For every word $a=a_{1} \ldots a_{n} \in W^{n}$ exactly one of the following holds. 
(a) There is exactly one sequence of maps $A^{1}, A^{2}, \ldots, A^{n-1}$ such that $a=A^{n-1} \cdots A^{1}(0)$ where the $A^{j}$ are maps of type $A_{\ell, a}^{j}$ as in Theorem 8.

(b) There is exactly one $k \leqslant n$ and exactly one sequence of maps $A^{k}, A^{k+1}, \ldots, A^{n-1}$ such that $a=A^{n-1} \cdots A^{k}\left(a^{\prime}\right)$ where the $A^{j}$ are maps of type $A_{\ell, a}^{j}$ as in Theorem 8 and $a^{\prime}=0 \ldots 012 \in W^{k}$.

Proof. The claim follows immediately from Theorem 8. Recall that $W^{n}$ is the disjoint union of the ranges of the seven maps given in Theorem 8 and $C_{3}^{n}=\{0 \ldots 012\}$. Therefore, every word $a \in W^{n}$ belongs either to $C_{3}^{n}$ or to the range of exactly one of the seven maps. If $a \in C_{3}^{n}$, then (b) holds with $k=n$. Otherwise there is exactly one map $A^{n-1}$ and, by the bijectivity of these maps, exactly one $a^{\prime} \in W^{n-1}$ such that $a=A^{n-1} a^{\prime}$. Now we repeat his process until we either fall in case (b) for some $1 \leqslant k<n$ or we reach the word 0 .

\section{Symplectic dual polar spaces}

For a symplectic space $(V, \omega)$ of dimension $2 n$ over the field with two elements $\mathbb{F}_{2}$, consider subspaces $U$ with $\omega(U)=0$, called totally isotropic. A subspace $U$ of $V$ is called a maximal totally isotropic subspace if it is totally isotropic and not properly contained in any other totally isotropic subspace of $V$. Every maximal totally isotropic subspace $X$ has dimension $n$. Every totally isotropic subspace $\widetilde{X}$ with $\operatorname{dim} \widetilde{X}=n-1$ is contained in exactly 3 different maximal totally isotropic subspaces. Moreover, every maximal isotropic subspace contains exactly $2^{n}-1$ totally isotropic subspaces of dimension $n-1$. We obtain a configuration of points and lines $\mathcal{G}_{n}:=\left(\mathcal{P}_{n}, \mathcal{L}_{n}\right)$, called the symplectic dual polar space, where

- $\mathcal{P}_{n}=$ the set of all maximal totally isotropic subspaces of $V$;

- $\mathcal{L}_{n}=$ the set of all totally isotropic subspaces of dimension $n-1$ of $V$.

In [BCN89, Lemma 9.4.1], Brouwer, Cohen and Neumaier give the following formula for the number of all maximal totally isotropic subspaces of a $2 n$-dimensional symplectic space:

$$
\left|\mathcal{P}_{n}\right|=\prod_{k=1}^{n}\left(2^{k}+1\right) \text { and }\left|\mathcal{L}_{n}\right|=\frac{\left(2^{n}-1\right)}{3} \prod_{k=1}^{n}\left(2^{k}+1\right)
$$

Every totally isotropic subspace $\widetilde{X}$ with $\operatorname{dim} \widetilde{X}=n-1$ is contained in exactly $p+1$ different maximal totally isotropic subspaces. We obtain a configuration of points and lines $\mathcal{G}_{n}(p):=\left(\mathcal{P}_{n}(p), \mathcal{L}_{n}(p)\right)$, where

$$
\left|\mathcal{P}_{n}(p)\right|=\sum_{k=0}^{n}\left[\left(\begin{array}{l}
n \\
k
\end{array}\right)_{p} p^{\frac{1}{2} k(k+1)}\right]=\prod_{k=1}^{n}\left(p^{k}+1\right) \text { and }\left|\mathcal{L}_{n}\right|=\frac{\left(p^{n}-1\right)}{p^{2}-1} \prod_{k=1}^{n}\left(p^{k}+1\right) .
$$

For $p=2$ we denote $\mathcal{G}_{n}:=\mathcal{G}_{n}(2)$. 
Definition 10. For the symplectic dual polar space $\mathcal{G}_{n}$ we define its collinearity graph $\Gamma$ as the graph whose vertices are the points of $\mathcal{P}_{n}$ and two vertices are adjacent if and only if the corresponding points are collinear in $\mathcal{G}_{n}$. This is also called the Menger graph, see $[$ Cox 50].

For example, $\mathcal{G}_{1}$ consists of one line and three points, so its collinearity graph is a triangle.

Definition 11. An embedding of $\mathcal{G}_{n}$ is an $\mathbb{F}_{2}$-vector space $E$ together with a map $\theta$ : $\mathcal{P}_{n} \rightarrow E$ such that

1. $\theta(P) \neq 0$ for every $P \in \mathcal{P}_{n}$,

2. $E=\operatorname{span}\{\operatorname{Rg}(\theta)\}$ where $\operatorname{Rg}(\theta)=\theta\left(\mathcal{P}_{n}\right)$ is the range of $\theta$.

3. $\theta(P)+\theta(Q)+\theta(R)=0$ for every line $L=\{P, Q, R\} \in \mathcal{L}_{n}$.

Such an embedding can be constructed as follows. Let $\mathbb{F}_{2}\left(\mathcal{L}_{n}\right)$ and $\mathbb{F}_{2}\left(\mathcal{P}_{n}\right)$ be the $\mathbb{F}_{2}$-vector spaces freely generated by the lines $\mathcal{L}_{n}$ and the points $\mathcal{P}_{n}$, respectively. Since every line $L \in \mathcal{L}_{n}$ can be written as $L=\{P, Q, R\}$ where $P, Q, R \in \mathcal{P}_{n}$ are the three points contained in $L$, we have the following map

$$
\eta: \mathbb{F}_{2}\left(\mathcal{L}_{n}\right) \longrightarrow \mathbb{F}_{2}\left(\mathcal{P}_{n}\right), \quad L=\{P, Q, R\} \mapsto P+Q+R .
$$

The quotient $U\left(\mathcal{G}_{n}\right):=\mathbb{F}_{2}\left(\mathcal{P}_{n}\right) / \eta\left(\mathbb{F}_{2}\left(\mathcal{L}_{n}\right)\right)$ is called the universal embedding module and we define the canonical map

$$
\theta: \mathcal{P}_{n} \longrightarrow U\left(\mathcal{G}_{n}\right) \text {. }
$$

Clearly this is an embedding of $\mathcal{G}_{n}$; it is called its universal embedding. Note that any other embedding is a quotient of the universal embedding. The dimension of the universal embedding of the polar dual space is $\operatorname{dim}\left(U\left(\mathcal{G}_{n}\right)\right)$.

Brouwer proved in 1990 that

$$
\operatorname{dim}\left(U\left(\mathcal{G}_{n}\right)\right) \geqslant \frac{\left(2^{n}+1\right)\left(2^{n-1}+1\right)}{3}
$$

and conjectured that (2) is in reality an equality. The conjecture was proved by Li [Li01] and independently by Blokhuis and Brouwer [BB03].

Theorem 12 ([Li01, BB03]). The dimension of the universal embedding of the polar dual space, $\operatorname{dim} U\left(\mathcal{G}_{n}\right)$, is

$$
\operatorname{dim} U\left(\mathcal{G}_{n}\right)=\frac{\left(2^{n}+1\right)\left(2^{n-1}+1\right)}{3} .
$$

Definition 13. Let $P$ and $Q$ be vertices in a connected graph $\Gamma$. A path from $P$ to $Q$ of length $n$ is an ordered set of vertices $V_{0}=P, V_{1}, \ldots, V_{n}=Q$ such that $V_{i-1}$ and $V_{i}$ are connected by an edge. The minimal length of all paths connecting $P$ and $Q$ is called the distance between $P$ and $Q$. 
Definition 14. Consider the collinearity graph $\Gamma$ associated to the symplectic dual polar space $\mathcal{G}_{n}$ and fix a vertex $X_{0}$ in the graph $\Gamma$. We denote by $\Gamma_{k}$ the induced subgraph formed by the vertices of $\Gamma$ which have distance $k$ from $X_{0}$.

It can be shown that all maximal isotropic subspaces $P, Q$ and $R$ of $V$ satisfy the following:

1. $\operatorname{dim}\left(X_{0} \cap P\right)=n-k$ if and only if $P \in \Gamma_{k}$.

2. $P$ and $Q$ belong to the same connected component of $\Gamma_{k}$ if and only if $X_{0} \cap P=$ $X_{0} \cap Q$.

3. The induced subgraph $\Gamma_{n}$ consists of exactly one connected component and $\Gamma_{1}$ consists of exactly $2^{n}-1$ disjoint connected components.

4. Suppose $P, Q, R$ are pairwise different and collinear. Then two of the spaces belong to the same $\Gamma_{k}$ and the third one belongs to $\Gamma_{k-1}$.

Example 15. Following [Li01], we write vectors $w \in V$ as row vectors $w=\left(w_{1}, \ldots, w_{2 n}\right)$ and for vectors $v_{1}, \ldots, v_{k} \in V$, we set $\left(\begin{array}{c}v_{1} \\ \vdots \\ v_{k}\end{array}\right):=\operatorname{span}\left\{v_{1}, \ldots, v_{k}\right\}$.

- Let $n=1$. Then $V=\mathbb{F}_{2}^{2}$ and its maximal isotropic subspaces are exactly the spans of the non-zero vectors of $V$. So we have $\mathcal{P}_{1}=\{(10),(01),(11)\}$ and $\mathcal{L}_{1}=\{(00)\}$, in particular $\operatorname{dim} U\left(\mathcal{G}_{1}\right)=2$.

- Let $n=2$. Then $V=\mathbb{F}_{2}^{4}$ and the elements of $\mathcal{P}_{2}$ are exactly the 15 following two-dimensional maximal isotropic subspaces:

$$
\begin{aligned}
& \left(\begin{array}{l}
1000 \\
0100
\end{array}\right), \quad\left(\begin{array}{l}
1000 \\
0101
\end{array}\right), \quad\left(\begin{array}{l}
1001 \\
0110
\end{array}\right), \quad\left(\begin{array}{l}
1001 \\
0111
\end{array}\right),\left(\begin{array}{l}
1010 \\
0100
\end{array}\right), \\
& \left(\begin{array}{l}
1010 \\
0101
\end{array}\right), \quad\left(\begin{array}{l}
1011 \\
0110
\end{array}\right), \quad\left(\begin{array}{l}
1011 \\
0111
\end{array}\right), \quad\left(\begin{array}{l}
1100 \\
0011
\end{array}\right),\left(\begin{array}{l}
1101 \\
0011
\end{array}\right), \\
& \left(\begin{array}{l}
1000 \\
0001
\end{array}\right), \quad\left(\begin{array}{l}
1010 \\
0001
\end{array}\right), \quad\left(\begin{array}{l}
0100 \\
0010
\end{array}\right), \quad\left(\begin{array}{l}
0101 \\
0010
\end{array}\right), \quad\left(\begin{array}{l}
0010 \\
0001
\end{array}\right) .
\end{aligned}
$$

The lines $\mathcal{L}_{2}$ are exactly the 15 following one-dimensional isotropic subspaces:

$$
\begin{array}{lllll}
(0001), & (0100), & (0111), & (1010), & (1101), \\
(0010), & (0101), & (1000), & (1011), & (1110), \\
(0011), & (0110), & (1001), & (1100), & (1111) .
\end{array}
$$

- Let $n=3$. Then $V=\mathbb{F}_{2}^{6}$ and the sets $\mathcal{P}_{3}$ and $\mathcal{L}_{3}$ consist of 135 point and 315 lines, respectively (see Appendix A). 
In the rest of this section we describe briefly how $\mathrm{Li}$ in [Li01] used certain vector spaces to count $\operatorname{dim} U\left(\mathcal{G}_{n}\right)$ and thereby proved Theorem 12 . Since (2) was already known, only the reverse inequality $\leqslant$ had to be proved.

To this end, Li considers the collinearity graph $\Gamma$ defined by $\mathcal{G}_{n}$. As before, we fix a point $X_{0}$ in $\Gamma$ and we set $\Gamma_{k}$ to be the set of all points in $\Gamma$ which have distance $k$ from $X_{0}$. Since every triangle in $\Gamma$ contains two elements from $\Gamma_{k}$ and one from $\Gamma_{k-1}$ for some $k=1, \ldots, n$, it follows that $\theta(Y) \in \operatorname{span}\left\{\theta\left(\Gamma_{k}\right)\right\}$ for every $Y \in \Gamma_{k-1}$. Thus we have the following filtration of $U\left(\mathcal{G}_{n}\right)$

$$
\{0\} \subset \operatorname{span}\left\{\theta\left(\Gamma_{0}\right)\right\}=\operatorname{span}\left\{\theta\left(X_{0}\right)\right\} \subset \operatorname{span}\left\{\theta\left(\Gamma_{1}\right)\right\} \subset \cdots \subset \operatorname{span}\left\{\theta\left(\Gamma_{n}\right)\right\}=U\left(\mathcal{G}_{n}\right)
$$

and consequently

$$
U\left(\mathcal{G}_{n}\right) \cong \operatorname{span}\left\{\theta\left(\Gamma_{0}\right)\right\} \oplus\left(\operatorname{span}\left\{\theta\left(\Gamma_{1}\right)\right\} / \operatorname{span}\left\{\theta\left(\Gamma_{0}\right)\right\}\right) \oplus \cdots \oplus\left(\operatorname{span}\left\{\theta\left(\Gamma_{n}\right)\right\} / \operatorname{span}\left\{\theta\left(\Gamma_{n-1}\right)\right\}\right) .
$$

Recall that two points $P, Q$ belong to the same connected component of $\Gamma_{k}$ if and only if $P \cap X_{0}=Q \cap X_{0}$. Clearly, this is the case if and only if $\theta(P) \equiv \theta(Q) \bmod \operatorname{span}\left\{\theta\left(\Gamma_{k-1}\right)\right\}$.

Now let $n \geqslant 3$ and $2 \leqslant k \leqslant n-1$ and let $L, M \subset X_{0}$ be subspaces with $\operatorname{dim} L=$ $n-k-1$ and $\operatorname{dim} M=n-k+2$. Then there are exactly 7 subspaces $L \subset R_{j} \subset M$ with $\operatorname{dim} R_{j}=n-k$, and $\sum_{j=1}^{7} \theta\left(\widetilde{R}_{j}\right) \equiv 0 \bmod \operatorname{span}\left\{\theta\left(\Gamma_{k-1}\right)\right\}$ where $\widetilde{R}_{j}$ is any maximal totally isotropic subspace of $V$ with $\widetilde{R}_{j} \cap X_{0}=R_{j}$.

For $1 \leqslant i \leqslant n$ we set $\mathcal{W}_{i}$ to be the $\mathbb{F}_{2}$-vector space freely generated by all $i$-dimensional subspaces of $X_{0}$ and for $1 \leqslant i<j \leqslant n$ we set $\mathcal{W}_{i j}$ to be the $\mathbb{F}_{2}$-vector space freely generated by all flags $X<Y$ in $X_{0}$ where $\operatorname{dim} X=i$ and $\operatorname{dim} Y=j$. Let $\left\{e_{L}\right\}$ be the natural basis of $\mathcal{W}_{i}$ and $\left\{e_{X<Y}\right\}$ the natural basis of $\mathcal{W}_{i j}$. Let us define the incidence map

$$
\phi_{n-k}: \mathcal{W}_{n-k-1, n-k+2} \rightarrow \mathcal{W}_{n-k}, \quad \phi_{n-k}\left(e_{X<Y}\right)=\sum_{\substack{X \subset L \subset Y \\ \operatorname{dim} L=n-k}} e_{L}
$$

Moreover, we have a natural surjection

$$
h_{n-k}: \mathcal{W}_{n-k} \rightarrow \operatorname{span}\left\{\theta\left(\Gamma_{k}\right)\right\} / \operatorname{span}\left\{\theta\left(\Gamma_{k-1}\right)\right\} .
$$

It follows from the above that $h_{n-k} \circ \phi_{n-k}=0$, hence the induced map

$$
\widetilde{h}_{n-k}: \mathcal{W}_{n-k} / \operatorname{Rg}\left(\phi_{n-k}\right) \rightarrow \operatorname{span}\left\{\theta\left(\Gamma_{k}\right)\right\} / \operatorname{span}\left\{\theta\left(\Gamma_{k-1}\right)\right\}
$$

is well-defined and surjective. Therefore

$$
\begin{aligned}
\operatorname{dim} U\left(\mathcal{G}_{n}\right)=\operatorname{dim} & \left.\left(\operatorname{span}\left\{\theta\left(\Gamma_{0}\right)\right\}\right\}\right)+\operatorname{dim}\left(\operatorname{span}\left\{\theta\left(\Gamma_{1}\right)\right\} / \operatorname{span}\left\{\theta\left(\Gamma_{0}\right)\right\}\right) \\
& +\sum_{k=2}^{n-1} \operatorname{dim}\left(\operatorname{span}\left\{\theta\left(\Gamma_{k}\right)\right\} / \operatorname{span}\left\{\theta\left(\Gamma_{k-1}\right)\right\}\right) \\
& +\operatorname{dim}\left(\operatorname{span}\left\{\theta\left(\Gamma_{n}\right)\right\} / \operatorname{span}\left\{\theta\left(\Gamma_{n-1}\right)\right\}\right)
\end{aligned}
$$




$$
\begin{aligned}
& \leqslant 1+\left(2^{n}-1\right)+\sum_{j=1}^{n-2} \operatorname{dim}\left(\mathcal{W}_{j} / \operatorname{Rg}\left(\phi_{j}\right)\right)+1 \\
& =1+2^{n}+\sum_{j=1}^{n-2} \operatorname{dim}\left(\mathcal{W}_{j}\right)-\operatorname{dim}\left(\operatorname{Rg}\left(\phi_{j}\right)\right) \\
& =\sum_{j=0}^{n} \operatorname{dim}\left(\mathcal{W}_{j}\right)-\sum_{j=1}^{n-2} \operatorname{dim}\left(\operatorname{Rg}\left(\phi_{j}\right)\right)
\end{aligned}
$$

where in the last step we have used that $\operatorname{dim} \mathcal{W}_{0}=\operatorname{dim} \mathcal{W}_{n}=1, \operatorname{dim} \mathcal{W}_{n-1}=2^{n}-1$.

In order to evaluate the right hand side, Li introduces the following order on $X_{0} \cong \mathbb{F}_{2}^{n}$. Let $e_{1}, \ldots, e_{n}$ be the standard basis of $\mathbb{F}_{2}^{n}$ and let $v=\alpha_{1} e_{1}+\cdots+\alpha_{n} e_{n}$ and $w=\beta_{1} e_{1}+$ $\cdots+\beta_{n} e_{n}$ in $\mathbb{F}_{2}^{n}$. Then we define the support and weight of $v$ as

$$
\operatorname{supp} v=\left\{j: \alpha_{j} \neq 0\right\}, \quad \text { wt } v=|\operatorname{supp} v|
$$

and we set $m(v)=\min (\operatorname{supp} v)$ and $M(v)=\max (\operatorname{supp} v)$. We obtain a total order on $\mathbb{F}_{2}^{n}$ by setting $v \succ w$ if and only if there is a $j=1, \ldots, n$ such that $\alpha_{k}=\beta_{k}$ for all $1 \leqslant k<j$ and $\left(\alpha_{j}, \beta_{j}\right)=(1,0)$.

If $L \subseteq \mathbb{F}_{2}^{n}$ is a subspace, we set $\operatorname{supp} L=\bigcup_{v \in L} \operatorname{supp} v$ and $m(L)=\{m(v): v \in$ $L\}$. It is not hard to see that $\operatorname{dim} L=|m(L)|$. The so-called reduced echelon basis of $L$ is the unique basis $v_{1}, \ldots, v_{k}$ such that $m\left(v_{j}\right)$ is strictly increasing and $m\left(v_{j}\right) \neq$ $\operatorname{supp}\left\{v_{1}, \ldots, v_{j-1}, v_{j+1}, \ldots, v_{k}\right\}$. This basis is obtained easily if we take an arbitrary basis of $L$, form the matrix whose rows are these basis vectors and apply the GaußJordan procedure to obtain a reduced row-echelon matrix. The rows of this new matrix form the reduced echelon basis of $L$. Now we define an order on the subspaces of $\mathbb{F}_{2}^{n}$ as follows. Let $L, L^{\prime}$ be subspaces of $\mathbb{F}_{2}^{n}$ with $\operatorname{dim} L=\operatorname{dim} L^{\prime}=k$ and reduced echelon basis $v_{1}, \ldots, v_{n}$ and $v_{1}^{\prime}, \ldots, v_{n}^{\prime}$ respectively. Then we say that $L \succ L^{\prime}$ if there is $j=1, \ldots, n$ such that $v_{k}=v_{k}^{\prime}$ for $k>j$ and $v_{j} \succ v_{j}^{\prime}$.

Recall that an element $\Delta \in \operatorname{Rg} \phi_{k}$ is a formal sum of $k$-dimensional subspaces of $X_{0}$. Let us set $A_{k}=\left\{\max \Delta: \Delta \in \operatorname{Rg}\left(\phi_{k}\right)\right\}$. Then it is not hard to see that $\operatorname{dim}\left(\operatorname{Rg}\left(\phi_{k}\right)\right)=$ $\left|A_{k}\right|$.

Now we define $\mathcal{N}^{n}:=\left\{L \subset X_{0}\right\} \backslash \bigcup_{k=1}^{n-2} A_{k}$ to be the set of subspaces of $X_{0}$ which belong to no $A_{k}$. Clearly, all the sets $A_{k}$ are disjoint. Recall that $\operatorname{dim} \mathcal{W}_{j}$ is the number of all subspaces of $X_{0}$ of dimension $j$. So we obtain from (3)

$$
\operatorname{dim} U\left(\mathcal{G}_{n}\right) \leqslant \sum_{j=0}^{n} \operatorname{dim}\left(\mathcal{W}_{j}\right)-\sum_{j=0}^{n}\left|A_{j}\right|=\left|\mathcal{N}^{n}\right| .
$$

$\mathrm{Li}$ gives a clever description of the elements in $\mathcal{N}^{n}$ using the reduced echelon basis as follows, see also [McC00]. 
Theorem 16. Let $L$ be a $k$-dimensional subset of $X_{0}$ with reduced echelon basis $v_{1} \succ$ $\cdots \succ v_{k}$. Then $L \in \mathcal{N}^{n}$ if and only if the following four conditions are satisfied:

(N1) wt $v_{j} \leqslant 2$ for all $j=1, \ldots, k$.

(N2) If $v_{r} \succ v_{s}$ and wt $v_{r}=$ wt $v_{s}=2$, then $M\left(v_{r}\right) \leqslant M\left(v_{s}\right)$.

(N3) If $v_{r} \succ v_{s} \succ v_{t}$, wt $v_{r}=$ wt $v_{s}=$ wt $v_{t}=2$ and $M\left(v_{r}\right)=M\left(v_{s}\right)<M\left(v_{t}\right)$, then $m\left(v_{t}\right)>M\left(v_{r}\right)$.

(N4) If $v_{r} \succ v_{s} \succ v_{t} \succ v_{u}$ and wt $v_{r}=\mathrm{wt} v_{s}=\mathrm{wt} v_{t}=\mathrm{wt} v_{u}=2$, then it is impossible that $M\left(v_{r}\right)=M\left(v_{s}\right)=M\left(v_{t}\right)<m\left(v_{u}\right)$.

Note that the last condition in (N4) is equivalent to $M\left(v_{r}\right)=M\left(v_{s}\right)=M\left(v_{t}\right)<M\left(v_{u}\right)$ by condition (N3). Then Li shows how $\mathcal{N}^{n+1}$ can be constructed from $\mathcal{N}^{n}$ and thus is able to show that

$$
\left|\mathcal{N}^{n}\right|=\frac{\left(2^{n}+1\right)\left(2^{n-1}+1\right)}{3},
$$

proving the formula in Theorem 12. In addition, it follows that the functions $\widetilde{h}_{n-k}$ are bijections and that $\operatorname{Rg}\left(\phi_{k}\right)=\operatorname{ker}\left(h_{n-k}\right)$.

If we modify slightly Li's construction of $\mathcal{N}^{n+1}$ from $\mathcal{N}^{n}$, then it is analogous to how we constructed $W^{n+1}$ from $W^{n}$. In the next section we will show how this allows us to construct a bijection between $W^{n+1}$ and $\mathcal{N}^{n}$.

\section{Bijection between words and vector spaces}

As in [Li01] we set $g(n):=\left|\mathcal{N}^{n}\right|$. Before we continue, let us give some examples of $\mathcal{N}^{n}$. We use the notation of Example 15.

- The set of all $L \in \mathcal{N}^{1}$ is (0) and (1) and $g(1)=\left|\mathcal{N}^{1}\right|=2=g_{W}(2)$.

- The set of all $L \in \mathcal{N}^{2}$ is, in ascending order, (00), (01), (10), (11), ( $\left.\begin{array}{l}10 \\ 01\end{array}\right)$ and $g(2)=$ $\left|\mathcal{N}^{2}\right|=5=g_{W}(3)$.

- The set of all $L \in \mathcal{N}^{3}$ is, in ascending order,

(000), (001), (010), (100), (011), (101), (110),

$$
\left(\begin{array}{l}
010 \\
001
\end{array}\right), \quad\left(\begin{array}{l}
100 \\
001
\end{array}\right), \quad\left(\begin{array}{l}
110 \\
001
\end{array}\right), \quad\left(\begin{array}{l}
100 \\
010
\end{array}\right), \quad\left(\begin{array}{l}
101 \\
010
\end{array}\right), \quad\left(\begin{array}{l}
100 \\
011
\end{array}\right), \quad\left(\begin{array}{l}
101 \\
011
\end{array}\right),\left(\begin{array}{l}
100 \\
010 \\
001
\end{array}\right)
$$

and $g(3)=\left|\mathcal{N}^{3}\right|=15=g_{W}(4)$. 
Now we show how $\mathcal{N}^{n}$ can be constructed from $\mathcal{N}^{n-1}$ for $n \geqslant 2$. For this to be analogous to the process for the passage from $W^{n}$ to $W^{n+1}$, we have to modify Li's procedure slightly.

In what follows, we identify vector spaces $L$ with the matrix $A$ whose rows consist of the reduced echelon basis of $L$ and we use the following notation: If $v \in \mathbb{F}_{2}^{n-1}$, then we denote by $\widetilde{v} \in \mathbb{F}_{2}^{n}$ the vector obtained from $v$ by appending a 0 and by $\widehat{v} \in \mathbb{F}_{2}^{n}$ the vector obtained from $v$ by inserting a 0 between the last and second to last component of $v$. The $k$ th unit vector in $\mathbb{F}_{2}^{n}$ is denoted by $e_{k}^{n}$.

We will say that a vector space $\widetilde{L} \in \mathcal{N}^{n}$ is in Case $k^{\prime}$ for $k^{\prime}=1, \ldots, 8$ if it is constructed from a vector space $L \in \mathcal{N}^{n-1}$ as described in the Cases $k^{\prime}$ below. For examples of these constructions, see the ones in Example 19.

- Case $\mathbf{1}^{\prime}$. Take an arbitrary vector space in $\mathcal{N}^{n-1}$ with reduced echelon basis $v_{1} \succ$ $\cdots \succ v_{k}$. Append 0 to each of these vectors in order to obtain $\widetilde{v}_{1} \succ \cdots \succ \widetilde{v}_{k}$. Then clearly $\widetilde{L} \in \mathcal{N}^{n}$ and $\operatorname{dim} L=\operatorname{dim} \widetilde{L}$. We denote this construction by

$$
\alpha_{n, 0}^{n-1}: \mathcal{N}^{n-1} \rightarrow \mathcal{N}^{n}, \quad L \mapsto \widetilde{L}
$$

Note that each vector space $\widetilde{L}$ obtained in this way has the form $\left(\begin{array}{cc}0 \\ L & \vdots \\ 0\end{array}\right)$ for some vector space $L \in \mathcal{N}^{n-1}$. The total number of such vector spaces $\widetilde{L}$ is $g(n-1)$.

- Case $2^{\prime}$. Take an arbitrary vector space in $\mathcal{N}^{n-1}$ with reduced echelon basis $v_{1} \succ$ $\cdots \succ v_{k}$. Append 0 to each of these vectors in order to obtain $\widetilde{v}_{1} \succ \cdots \succ \widetilde{v}_{k}$ and augment this basis by $e_{n}^{n}$ to a reduced echelon basis of $\widetilde{L}:=\operatorname{span}\left\{\widetilde{v}_{1}, \ldots, \widetilde{v}_{k}, e_{n}^{n}\right\}$. Then clearly $\widetilde{L} \in \mathcal{N}^{n}$ and $\operatorname{dim} \widetilde{L}=\operatorname{dim} L+1$. We denote this construction by

$$
\alpha_{n, 1}^{n-1}: \mathcal{N}^{n-1} \rightarrow \mathcal{N}^{n}, \quad L \mapsto \widetilde{L}
$$

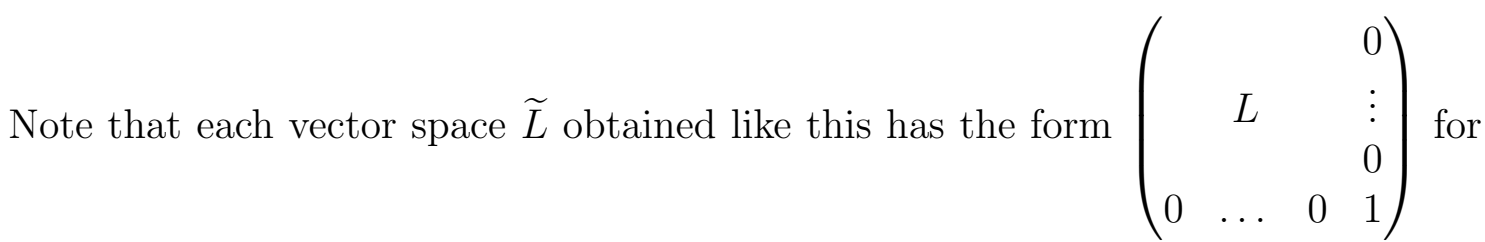
some vector space $L \in \mathcal{N}^{n-1}$. The total number of such vector spaces $\widetilde{L}$ is $g(n-1)$.

By construction, every vector space obtained in Cases 1' and 2' has either only zeros in the last column or its last line is the vector $e_{n}^{n}$.

For the remaining cases $3^{\prime}, 4^{\prime}, 5^{\prime}, 6^{\prime}$ and $7^{\prime}$ we take an arbitrary vector space $L \in \mathcal{N}^{n-1}$ with reduced echelon basis $v_{1} \succ \cdots \succ v_{k}$ such that $n-1 \in \operatorname{supp} L$ and $v_{k} \neq e_{n-1}^{n-1}$. This means that the matrix consisting of the row vectors $v_{1}, \ldots, v_{k}$ has at least one 1 in its last column and the last row is not equal to $e_{n-1}^{n-1}$ and $L$ cannot have been obtained from (0) or $(1) \in \mathcal{N}^{1}$ by using only the constructions described in Cases $1^{\prime}$ and $2^{\prime}$. 
- Case $3^{\prime}$. Insert a 0 in front of the last coordinate of each of the basis vectors in order to obtain $\widehat{v}_{1} \succ \cdots \succ \widehat{v}_{k}$ and set $\widetilde{L}:=\operatorname{span}\left\{\widehat{v}_{1}, \ldots, \widehat{v}_{k}\right\}$. We denote this construction by

$$
\alpha_{n-1,0}^{n-1}: L \mapsto \widetilde{L}
$$

Clearly $\widetilde{L} \in \mathcal{N}^{n}$ and $\operatorname{dim} \widetilde{L}=\operatorname{dim} L$.

- Case $4^{\prime}$. As in Case $3^{\prime}$, insert a 0 in front of the last coordinate of each of the basis vectors in order to obtain $\widehat{v}_{1} \succ \cdots \succ \widehat{v}_{k}$ and set $\widetilde{L}:=\operatorname{span}\left\{\widehat{v}_{1}, \ldots, \widehat{v}_{k}, e_{n-1}^{n}\right\}$. We denote this construction by

$$
\alpha_{n-1,1}^{n-1}: L \mapsto \widetilde{L}
$$

Clearly $\widetilde{L} \in \mathcal{N}^{n}$ and $\operatorname{dim} \widetilde{L}=\operatorname{dim} L+1$.

- Case $5^{\prime}$. As in Case $3^{\prime}$, insert a 0 in front of the last coordinate of each of the basis vectors in order to obtain $\widehat{v}_{1} \succ \cdots \succ \widehat{v}_{k}$ and set $\widetilde{L}:=\operatorname{span}\left\{\widehat{v}_{1}, \ldots, \widehat{v}_{k}, e_{n-1}^{n}+e_{n}^{n}\right\}$. We denote this construction by

$$
\alpha_{n-1,2}^{n-1}: L \mapsto \widetilde{L}
$$

Clearly $\widetilde{L} \in \mathcal{N}^{n}$ and $\operatorname{dim} \widetilde{L}=\operatorname{dim} L+1$.

The number of vector spaces in each of the Cases $3^{\prime}, 4^{\prime}, 5^{\prime}$ is

$$
g(n-1)-\#\left(L \in \mathcal{N}^{n-1} \text { with last column zero or } v_{k}=e_{n-1}^{n-1}\right)=g(n-1)-2 g(n-2)
$$

because $\#\left(L \in \mathcal{N}^{n-1}\right.$ with last column zero $)=\#\left(L \in \mathcal{N}^{n-1}\right.$ with $\left.v_{k}=e_{n-1}^{n-1}\right)=g(n-2)$ as in Case $1^{\prime}$.

- Case $6^{\prime}$. Let $L \in \mathcal{N}^{n-1}$ with reduced echelon basis $v_{1} \succ \cdots \succ v_{k}$ such that either the last column of $A$ has at least two ones (this corresponds to case 6 in [Li01]), or such that the last column has exactly one 1 and that this 1 is not in the first row (this is a subset of the cases $7 \mathrm{~b}, \mathrm{c}$ and $\mathrm{d}$ of [Li01]).

- Case 6'a. Assume that the last column of $A$ has at least two ones. Then every row with a 1 in its last column must have weight 2 . Set $j=\min \left\{\ell: M\left(v_{\ell}\right)=\right.$ $n-1\}=$ highest row of $A$ with a 1 in the last column. Let $b<n-1$ such that $v_{j}=e_{b}^{n-1}+e_{n-1}^{n-1}$. For $\ell \neq j$ we let $\widehat{v}_{\ell}$ be the vector in $\mathbb{F}^{n}$ which is obtained from $v_{\ell}$ by inserting a 0 between the last and the second to last component of $v_{\ell}$. Then we set $\widetilde{L}=\operatorname{span}\left\{\widehat{v}_{1}, \ldots, \widehat{v}_{j-1}, e_{b}^{n-1}+e_{n-1}^{n-1}, \widehat{v}_{j+1}, \ldots, \widehat{v}_{k}\right\}$. In words: we append to $A$ a zero column and then we push the 1 s in the $(n-1)$ th column below the $j$ th row out to the new $n$th column. The matrix $A^{\prime}$ corresponding to $\widetilde{L}$ has exactly one 1 in the second to last column; this occurs in a row with weight 2 , different from the last row and there is at least one row of the form $e_{a}^{n}+e_{n}^{n}$ with $a>j$. Note that $\operatorname{dim} \widetilde{L}=\operatorname{dim} L$. This is the case 6 in [Li01]. 
- Case $\mathbf{6}^{\prime} \mathbf{b}$. Assume that the last column of $A$ has at exactly one 1 and let $j>2$ such that $v_{j}=e_{a}^{n-1}+e_{n-1}^{n-1}$. Let $b=\max \left\{M\left(v_{\ell}\right): \ell \neq j\right\}$.

If $b<a$, then necessarily $j=k$ and we set $\widetilde{L}=\operatorname{span}\left\{\widehat{v}_{1}, \ldots, \widehat{v}_{k-1}, e_{a}^{n}, e_{n-1}^{n}+\right.$ $\left.e_{n}^{n}\right\}$ (this is part of case 7a in [Li01]). Note that $\operatorname{dim} \widehat{L}=\operatorname{dim} L+1$.

If $a<b<n-1$ and there exists $\ell>j$ with $b \in \operatorname{supp} v_{\ell}$, then $v_{\ell}=v_{k}=$ $e_{b}^{n-1}$ and $b \notin \operatorname{supp} v_{m}$ for $m \neq \ell$. We set $\widetilde{L}=\operatorname{span}\left\{\widehat{v}_{1}, \ldots, \widehat{v}_{j-1}, e_{a}^{n}+\right.$ $\left.e_{b}^{n}, \widehat{v}_{j+1}, \ldots, \widehat{v}_{k-1}, e_{n-1}^{n}+e_{n}^{n}\right\}$ (this is part of case $7 \mathrm{~b}$ in [Li01]). Note that $\operatorname{dim} \widetilde{L}=\operatorname{dim} L$.

If $a<b<n-1$ and there exists $\ell<j$ with $b \in \operatorname{supp} v_{\ell}$, then wt $v_{\ell}=2$ and there is $c<a<b$ such that $v_{\ell}=e_{c}^{n-1}+e_{b}^{n-1}$. We set $\widetilde{L}=\operatorname{span}\left\{\widehat{v}_{1}, \ldots, \widehat{v}_{j-1}, e_{a}^{n}+\right.$ $\left.e_{b}^{n}, \widehat{v}_{j+1}, \ldots, \widehat{v}_{k-1}, \widehat{v}_{k}, e_{n-1}^{n}+e_{n}^{n}\right\}$ (this is case $7 \mathrm{c}$ in [Li01]). Note that $\operatorname{dim} \widetilde{L}=$ $\operatorname{dim} L+1$.

We denote these constructions by

$$
\alpha_{n-1,3}^{n-1}: L \mapsto \widetilde{L}
$$

- Case $7^{\prime}$. Let $L \in \mathcal{N}^{n-1}$ with reduced echelon basis $v_{1} \succ \cdots \succ v_{k}$ such that $L$ is not in case 6'. Then wt $v_{1}=2, n-1 \in \operatorname{supp} v_{1}$ and $n-1 \notin \operatorname{supp} v_{j}$ for $j \geqslant 2$. That implies that wt $v_{j}=1$ for $j \geqslant 2$. Moreover, it implies that $L$ originates from a vector space $\widehat{L} \in \mathcal{N}^{\widehat{n}}$ in Case 8 using only the constructions described in Cases $3^{\prime}$ and $4^{\prime}$.

Let $v_{1}=e_{b}^{n-1}+e_{n-1}^{n-1}$. If $\operatorname{dim} L=1$, we set $\widetilde{L}=\operatorname{span}\left\{e_{b}^{n}, e_{n-1}^{n}+e_{n}^{n}\right\}$ (this corresponds to 1-dimensional vector spaces of case $7 \mathrm{a}$ in $[\mathrm{Li01}])$.

If $\operatorname{dim} L>1$ and $v_{k}=e_{a}^{n-1}$, we set $\widetilde{L}=\operatorname{span}\left\{e_{b}^{n}+e_{a}^{n}, \widehat{v}_{2}, \ldots, \widehat{v}_{k-1}, e_{n-1}^{n}+e_{n}^{n}\right\}$ (this corresponds to some of the vector spaces of case $7 \mathrm{~b}$ in [Li01]).

We denote these constructions by

$$
\alpha_{n, 3}^{n-1}: L \mapsto \widetilde{L} .
$$

The total number of vector spaces in the Cases $6^{\prime}$ and $7^{\prime}$ together is

$$
g(n-1)-\#\left(L \in \mathcal{N}^{n-1} \text { with last column zero or } v_{k}=e_{n-1}^{n-1}\right)=g(n-1)-2 g(n-2) .
$$

- Case $\mathbf{8}^{\prime}$. Let $\widetilde{L}=(0 \cdots 011) \in \mathcal{N}^{n}$. Clearly this vector space is not contained in the spaces constructed so far.

It is not hard to see that the vector spaces constructed above are all pairwise disjoint and that they all belong to $\mathcal{N}^{n}$. Moreover, it can be seen that we obtain every $\widetilde{L} \in \mathcal{N}^{n}$ in exactly one way.

It is clear that the cases $k$ for words and $k^{\prime}$ for vector spaces correspond to each other. E.g., appending a 0 to a given word corresponds to appending a zero column to a vector space (Case 1 and $1^{\prime}$ ); appending a 1 to a given word corresponds to appending a zero column to a vector space and adding the base vector $x_{n}$ (Case 2 and $2^{\prime}$ ); etc.

Therefore we obtain the following theorem in analogy to Theorem 9: 
Theorem 17. Let $n \geqslant 2$. Then for every vector space $L \in \mathcal{N}^{n}$ exactly one of the following holds.

(a) There is exactly one sequence of maps $B^{2}, \ldots, B^{n-1}$ such that $L=B^{n-1} \cdots B^{2}(0)$ or $L=B^{n-1} \cdots B^{2}(1)$ where the $B^{j}$ are maps of type $\alpha_{\ell, a}^{j}$ as in the cases above.

(b) There is exactly one $k \leqslant n$ and exactly one sequence of maps $B^{k}, B^{k+1}, \ldots, B^{n-1}$ such that $L=B^{n-1} \cdots B^{k}(\widehat{L})$ where the $B^{j}$ are maps of type $\alpha_{\ell, a}^{j}$ as in the cases above and $\widehat{L}=(0 \ldots 011) \in \mathcal{N}^{k}$.

Proof. From what we just saw, $\mathcal{N}^{n}$ is the disjoint union of $\{(0 \cdots 011)\}$ and the ranges of the seven maps $\alpha_{n, 0}^{n-1}, \alpha_{n, 1}^{n-1}, \alpha_{n-1,0}^{n-1}, \alpha_{n-1,1}^{n-1}, \alpha_{n-1,2}^{n-1}, \alpha_{n-1,3}^{n-1}, \alpha_{n, 3}^{n-1}$. Now the proof is essentially the same as the proof of Theorem 9: Given a vector space $L \in \mathcal{N}^{n}$, it is either equal to $(0 \cdots 011)$ or it belongs to the range of exactly one of the seven maps above. If $L=(0 \cdots 011)$, then (b) holds with $k=n$. Otherwise there is exactly one map $B^{n-1}$ among the maps above and, by the bijectivity of these maps, exactly one $L^{\prime} \in \mathcal{N}^{n-1}$ such that $L=B^{n-1} L^{\prime}$. Now we repeat his process until we either fall in case (b) for some $1 \leqslant k<n$ or we reach the vector space (0) or (1).

Now Theorem 9 and Theorem 17 together give a bijection between $W^{n+1}$ and $\mathcal{N}^{n}$.

Theorem 18. Let $n \in \mathbb{N}$. We have the following bijection

$$
\Psi: W^{n+1} \rightarrow \mathcal{N}^{n}
$$

defined as follows.

1. $\Psi(00)=(0), \Psi(01)=(1)$.

2. If $a=0 \ldots 012 \in W^{n+1}$, we set $\Psi(a)=(0 \ldots 011) \in \mathcal{N}^{n}$.

3. If $a=A^{n-1} \cdots A^{2}(00)$, we set $L=B^{n-1} \cdots B^{2}(0)$.

4. If $a=A^{n-1} \cdots A^{2}(01)$, we set $L=B^{n-1} \cdots B^{2}(1)$.

5. If $a=A^{n-1} \cdots A^{k}(0 \ldots 012)$, we set $L=B^{n-1} \ldots B^{k}(0 \ldots 011)$

where we use the correspondence $A_{n+1, j}^{n} \longleftrightarrow \alpha_{n, j}^{n-1}$ for $j=0,1, \quad A_{n, j}^{n} \longleftrightarrow \alpha_{n-1, j}^{n-1}$ for $j=0,1,2,3$, and $A_{n+1,3}^{n} \longleftrightarrow \alpha_{n, 3}^{n-1}$. In particular, $\left|\mathcal{N}^{n}\right|=g_{W}(n+1)$ and

$$
\operatorname{dim} U\left(\mathcal{G}_{n}\right)=\frac{\left(2^{n}+1\right)\left(2^{n-1}+1\right)}{3} .
$$

Proof. From Theorem 9 and the results in this section it is clear that $\Psi$ is a bijection, in particular it follows that $\left|\mathcal{N}^{n}\right|=g_{W}(n+1)=\frac{\left(2^{n}+1\right)\left(2^{n-1}+1\right)}{3}$. Therefore, the formula for $\operatorname{dim} U\left(\mathcal{G}_{n}\right)$ follows from $(2)$ and $(4)$. 


\section{Example 19.}

- $\Psi(01010010)=\left(\begin{array}{l}1000000 \\ 0010000 \\ 0000010\end{array}\right)$.

- $\Psi(0123210)=\left(\begin{array}{l}101000 \\ 010100 \\ 000010\end{array}\right)$ as the following diagram shows:

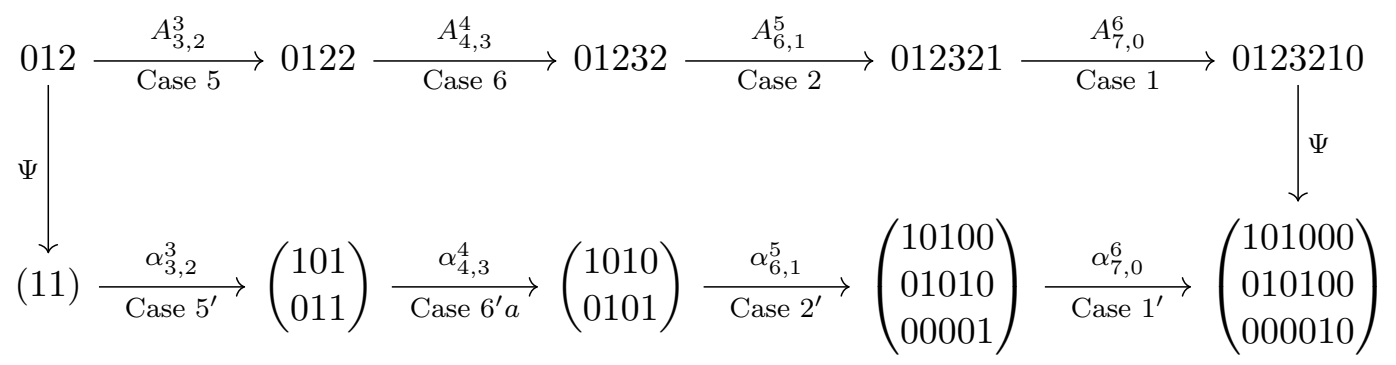

- $\Psi(00122333)=\left(\begin{array}{l}0100000 \\ 0010100 \\ 0001100 \\ 0000011\end{array}\right)$ as the following diagram shows:

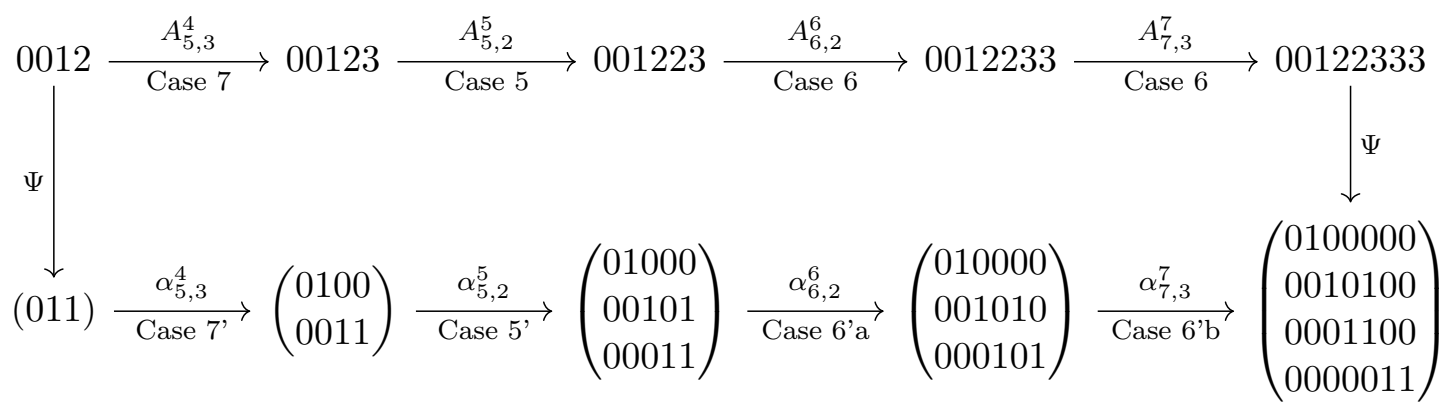

We give some examples of preimages of vector spaces (they are the spaces listed in [Li01] on page 105).

- $\Psi^{-1}\left(\left(\begin{array}{c}10001 \\ 01000 \\ 00011\end{array}\right)\right)=011022$ because 


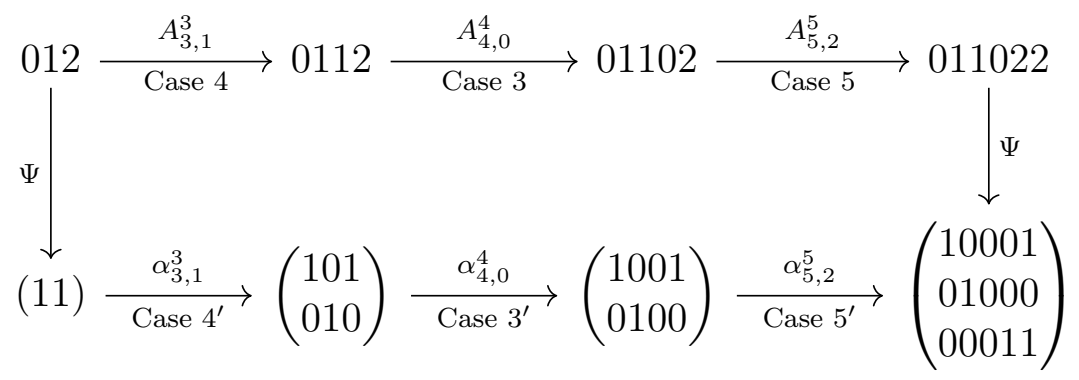

- $\Psi^{-1}\left(\left(\begin{array}{l}10010 \\ 01001 \\ 00101\end{array}\right)\right)=012232$ because

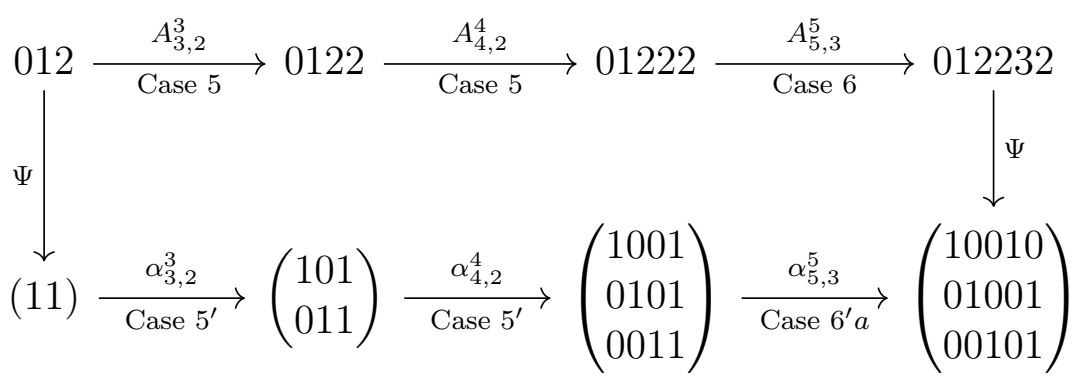

- $\Psi^{-1}\left(\left(\begin{array}{l}1000010 \\ 0100001 \\ 0010001 \\ 0001000 \\ 0000101\end{array}\right)\right)=01221232$ because

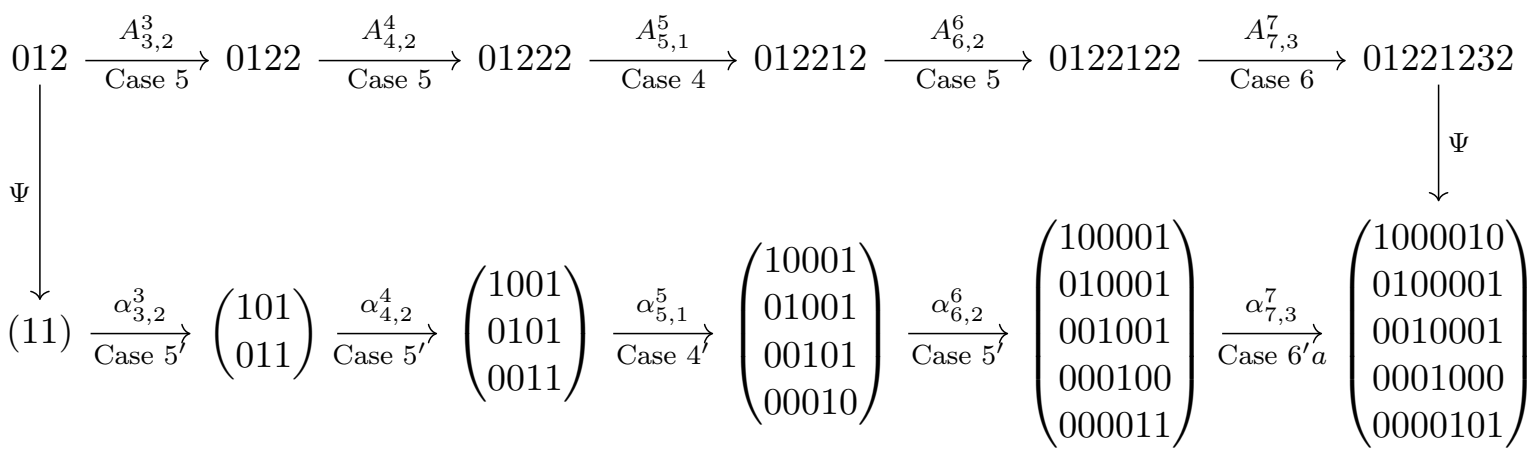

\section{A Symplectic Dual Polar Space for $n=1,2,3$}

Recall that $\mathcal{G}_{n}$ is the symplectic dual polar space defined in Section 3. It consists of $\prod_{k=1}^{n}\left(2^{k}+1\right)$ points and $\frac{1}{3}\left(2^{n}-1\right) \prod_{k=1}^{n}\left(2^{k}+1\right)$ lines. Each line contains exactly three points and through each point pass exactly $2^{n}-1$ lines because any $n$-dimensional $\mathbb{F}_{2^{-}}$ vector space contains exactly $2^{n}-1$ different $(n-1)$-dimensional subspaces. 


\section{A.1 $n=1$}

$\mathcal{G}_{1}$ consists of one line with exactly three points.

\section{A.2 $n=2$}

For $\mathcal{G}_{2}$ we label the 15 points by

$$
\begin{aligned}
& 0 \leftrightarrow\left(\begin{array}{c}
1000 \\
0100
\end{array}\right), \quad 1 \leftrightarrow\left(\begin{array}{c}
1000 \\
0101
\end{array}\right), \quad 2 \leftrightarrow\left(\begin{array}{c}
1001 \\
0110
\end{array}\right), \quad 3 \leftrightarrow\left(\begin{array}{c}
1001 \\
0111
\end{array}\right), \quad 4 \leftrightarrow\left(\begin{array}{c}
1010 \\
0100
\end{array}\right), \\
& 5 \leftrightarrow\left(\begin{array}{c}
1010 \\
0101
\end{array}\right), \quad 6 \leftrightarrow\left(\begin{array}{c}
1011 \\
0110
\end{array}\right), \quad 7 \leftrightarrow\left(\begin{array}{c}
1011 \\
0111
\end{array}\right), \quad 8 \leftrightarrow\left(\begin{array}{c}
1100 \\
0011
\end{array}\right), \quad 9 \leftrightarrow\left(\begin{array}{c}
1101 \\
0011
\end{array}\right), \\
& 10 \leftrightarrow\left(\begin{array}{c}
1000 \\
0001
\end{array}\right), \quad 11 \leftrightarrow\left(\begin{array}{c}
1010 \\
0001
\end{array}\right), \quad 12 \leftrightarrow\left(\begin{array}{c}
0100 \\
0010
\end{array}\right), \quad 13 \leftrightarrow\left(\begin{array}{c}
0101 \\
0010
\end{array}\right), \quad 14 \leftrightarrow\left(\begin{array}{c}
0010 \\
0001
\end{array}\right) .
\end{aligned}
$$

We used SageMath $\left[\mathrm{S}^{+} 09\right]$ to write down all lines as triples of their points:

$$
\begin{array}{lllll}
(0,1,10), & (6,7,11), & (3,4,9), & (1,5,13), & (12,13,14), \\
(2,3,10), & (0,7,8), & (2,5,8), & (2,6,12), & (8,9,14), \\
(4,5,11), & (1,6,9), & (0,4,12), & (3,7,13), & (10,11,14) .
\end{array}
$$

We fix the vertex 0 and construct the corresponding subgraphs $\Gamma_{0}, \Gamma_{1}$ and $\Gamma_{2}$.

- $\Gamma_{0}$ consists only of the vertex 0 .

- $\Gamma_{1}$ consists of three connected components, each of which contains two vertices and one edge.

- $\Gamma_{2}$ consists of one connected component and eight points which form a cube.

The subgraphs $\Gamma_{1}$ and $\Gamma_{2}$ are shown in Figure 2.

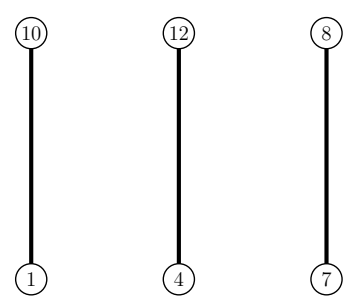

Subgraph $\Gamma_{1}$ of $\mathcal{G}_{2}$ consisting of three connected components.



Subgraph $\Gamma_{2}$ of $\mathcal{G}_{2}$ consisting of one connected component.

Figure 2: Induced subgraphs $\Gamma_{1}$ and $\Gamma_{2}$ of $\mathcal{G}_{2}$. 


\section{A.3 $n=3$}

The dual polar space $\mathcal{G}_{3}$ consists of 135 points and 315 lines. Each line contains exactly three points and through each point pass exactly seven lines. The subgraphs $\Gamma_{0}, \Gamma_{1}, \Gamma_{2}$ and $\Gamma_{3}$ have the following description:

- $\Gamma_{0}$ consists only of the vertex 0 .

- $\Gamma_{1}$ has seven connected components each of which consists of two vertices and one edge.

- $\Gamma_{2}$ consists of seven components each one with the form of a cube.

- $\Gamma_{3}$ is connected and consists of 64 vertices and 224 edges.

\section{B Construction of $W^{n+1}$ from $W^{n}$}

Let $n \geqslant 2$. In this appendix we show how $W^{n+1}$ is constructed from $W^{n}$. Recall that if $a=a_{1} a_{2} \ldots a_{n-1} a_{n} \in W^{n}$, then we can do the following (cf. Remark 6):

- If $a_{n} \in\{0,1\}$ : append 0 or 1 . We obtain a word in Case 1 or 2 .

- If $a_{n} \in\{2,3\}$ : insert 0,1 or 2 before $a_{n}$. We obtain a word in Case 3, 4 or 5 .

- If $a_{n} \in\{2,3\}$ and it is possible to insert 3 before $a_{n}$, we do so. We obtain a word in Case 6.

- If $a_{n} \in\{2,3\}$ and it is not possible to insert 3 before $a_{n}$, then necessarily $a_{n}=2$ and $a_{j} \in\{0,1\}$ for $1 \leqslant j \leqslant n-1$. We append 3 and obtain a word in Case 7 .

Finally, we have to add the word $a=0 \ldots 012$ from Case 8. In this way we obtain all possible words of $W^{n+1}$. 

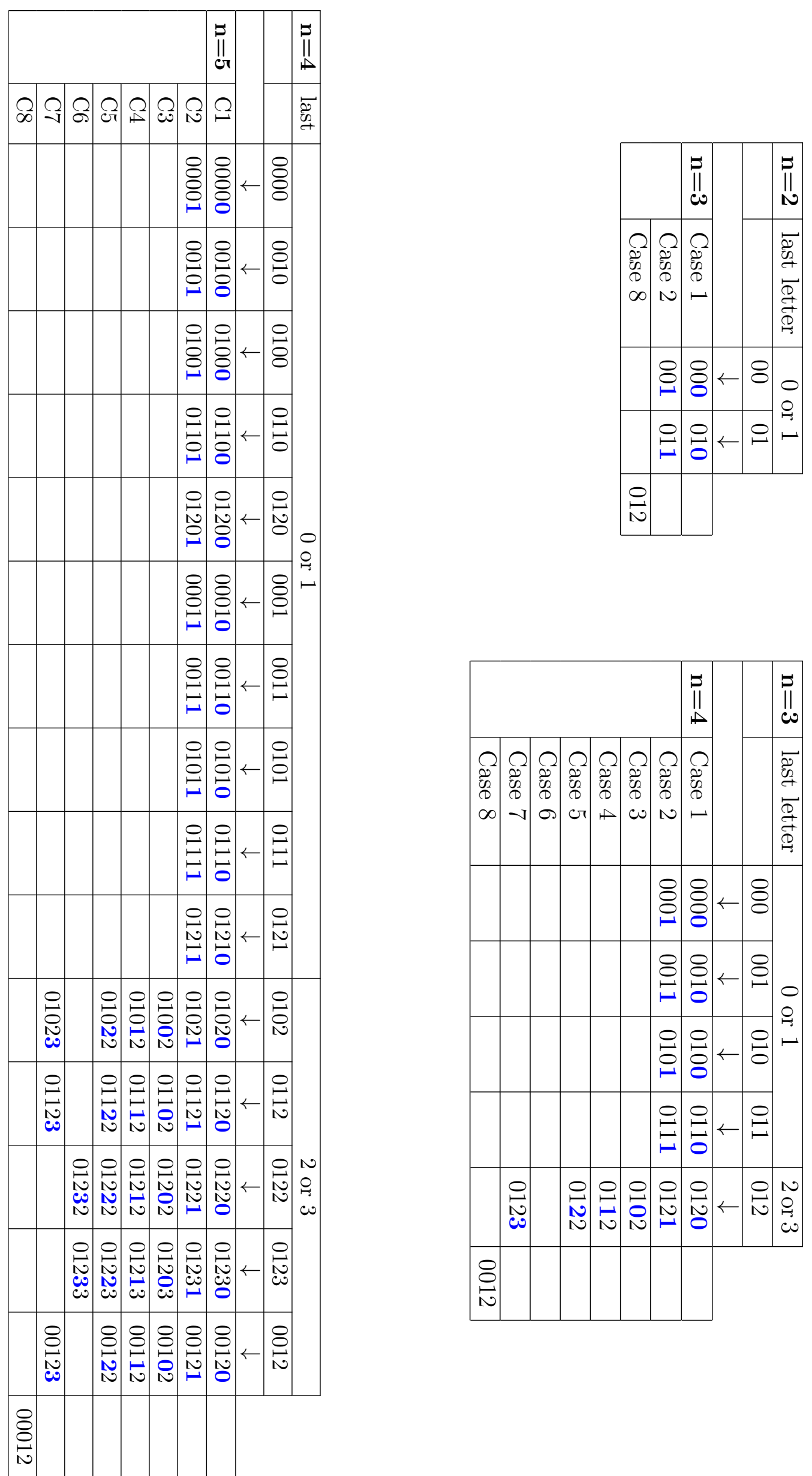


\section{Classification of words in $W^{n}$ according to the Cases $1-8$}

Let $n \geqslant 2$ and $a=a_{1} a_{2} \ldots a_{n-1} a_{n} \in W^{n}$. As before, we set $E_{n-1}^{n}(a)=a_{1} a_{2} \ldots a_{n-2} a_{n}$ which is obtained from $a$ by erasing its second to last letter. Recall that the word $a$ belongs to

- Case 1 if $a_{n}=0$;

- Case 2 if $a_{n}=1$;

- Case 3 if $a_{n} \in\{2,3\}$ and $a_{n-1}=0$ (then automatically $E_{n-1}^{n}(a) \in W^{n-1}$ );

- Case 4 if $a_{n} \in\{2,3\}, a_{n-1}=1$ and $E_{n-1}^{n}(a) \in W^{n-1}$;

- Case 5 if $a_{n} \in\{2,3\}, a_{n-1}=2$ and $E_{n-1}^{n}(a) \in W^{n-1}$;

- Case 6 if $a_{n} \in\{2,3\}$ and $a_{n-1}=3$ (then automatically $E_{n-1}^{n}(a) \in W^{n-1}$ );

- Case 7 if $a_{n} \in\{2,3\}, a_{n-1}=2$ and $E_{n-1}^{n}(a) \notin W^{n-1}$

(equivalently: if $a_{n-1} a_{n}=23$ and $a_{j} \in\{0,1\}$ for $1 \leqslant j \leqslant n-2$ );

- Case 8 if $a_{n} \in\{2,3\}, a_{n-1}=1$ and $E_{n-1}^{n}(a) \notin W^{n-1}$

(equivalently: if $a=0 \ldots 012$ ).

So we obtain for $n=1,2,3,4,5$ :

\begin{tabular}{|c|c|c|c|c|c|c|c|c|}
\hline \multirow[t]{2}{*}{$n=1$} & Case 1 & Case 2 & Case 3 & Case 4 & Case 5 & Case 6 & Case 7 & Case 8 \\
\hline & $\overline{0}$ & & & & & & & \\
\hline \multirow[t]{2}{*}{$n=2$} & Case 1 & Case 2 & Case 3 & Case 4 & Case 5 & Case 6 & Case 7 & Case 8 \\
\hline & 00 & 01 & & & & & & \\
\hline \multirow[t]{2}{*}{$n=3$} & Case 1 & Case 2 & Case 3 & Case 4 & Case 5 & Case 6 & Case 7 & Case 8 \\
\hline & $\begin{array}{l}000 \\
010\end{array}$ & $\begin{array}{l}001 \\
011\end{array}$ & & & & & & 012 \\
\hline
\end{tabular}

\begin{tabular}{|c|c|c|c|c|c|c|c|c|}
\hline$n=3$ & Case 1 & Case 2 & Case 3 & Case 4 & Case 5 & Case 6 & Case 7 & Case 8 \\
\hline & 0000 & 0001 & 0102 & 0112 & 0122 & & 0123 & 0012 \\
\hline & 0010 & 0011 & & & & & & \\
\hline & 0100 & 0101 & & & & & & \\
\hline & 0110 & 0111 & & & & & & \\
\hline & 0120 & 0121 & & & & & & \\
\hline
\end{tabular}




\begin{tabular}{|c|c|c|c|c|c|c|c|c|}
\hline$n=3$ & Case 1 & Case 2 & Case 3 & Case 4 & Case 5 & Case 6 & Case 7 & Case 8 \\
\hline & 00000 & 00001 & 01002 & 01012 & 01022 & \multirow{15}{*}{$\begin{array}{l}01232 \\
01233\end{array}$} & 01023 & \multirow[t]{15}{*}{00012} \\
\hline & 00100 & 00101 & 01102 & 01112 & 01122 & & 01123 & \\
\hline & 01000 & 01001 & 01202 & 01212 & 01222 & & & \\
\hline & 01100 & 01101 & 01203 & 01213 & 01223 & & & \\
\hline & 01200 & 01201 & 00102 & 00112 & 00122 & & 00123 & \\
\hline & 00010 & 00011 & & & & & & \\
\hline & 00110 & 00111 & & & & & & \\
\hline & 01010 & 01011 & & & & & & \\
\hline & 01110 & 01111 & & & & & & \\
\hline & 01210 & 01211 & & & & & & \\
\hline & 01020 & 01021 & & & & & & \\
\hline & 01120 & 01121 & & & & & & \\
\hline & 01220 & 01221 & & & & & & \\
\hline & 01230 & 01231 & & & & & & \\
\hline & 00120 & 00121 & & & & & & \\
\hline
\end{tabular}

\section{References}

[AS16] J. Arndt and N. J. A. Sloane. Counting Words that are in "Standard Order". OEIS, 2016. https://oeis.org/A278984/a278984.txt.

[Bak10a] H. F. Baker. Principles of geometry. Volume 2. Plane geometry., volume 103 of Cambridge Library Collection. Cambridge University Press, Cambridge, 2010 .

[Bak10b] H. F. Baker. Principles of geometry. Volume 4. Higher geometry., volume 103 of Cambridge Library Collection. Cambridge University Press, Cambridge, 2010 .

[BB03] A. Blokhuis and A. E. Brouwer. The universal embedding dimension of the binary symplectic dual polar space. Discrete Math., 264(1-3):3-11, 2003. The $2000 \mathrm{Com}^{2} \mathrm{MaC}$ Conference on Association Schemes, Codes and Designs (Pohang).

[BC13] F. Buekenhout and A. M. Cohen. Diagram Geometry. Related to classical groups and buildings. Ergebnisse der Mathematik und ihrer Grenzgebiete. 3. Folge. A Series of Modern Surveys in Mathematics [Results in Mathematics and Related Areas. 3rd Series. A Series of Modern Surveys in Mathematics], 57. Springer, Heidelberg, 2013.

[BCN89] A. E. Brouwer, A. M. Cohen, and A. Neumaier. Distance-regular graphs, volume 18 of Ergebnisse der Mathematik und ihrer Grenzgebiete (3) [Results in Mathematics and Related Areas (3)]. Springer-Verlag, Berlin, 1989. 
[BRRZ08] N. Bergeron, C. Reutenauer, M. Rosas, and M. Zabrocki. Invariants and coinvariants of the symmetric groups in noncommuting variables. Canad. J. Math., 60(2):266-296, 2008.

[Bru06] B. De Bruyn. Near Polygons. Frontiers in Mathematics. Birkhäuser Verlag, Basel, 2006.

[BS74] F. Buekenhout and E. Shult. On the foundations of polar geometry. Geometriae Dedicata, 3:155-170, 1974.

[Cam82] P. J. Cameron. Dual polar spaces. Geom. Dedicata, 12(1):75-85, 1982.

[Cox50] H. S. M. Coxeter. Self-dual configurations and regular graphs. Bull. Amer. Math. Soc., 56:413-455, 1950.

[Cre77] L. Cremona. Teoremi stereometrici dal quali si deducono le proprietà dell' esagrammo di Pascal. Reale Accad. dei Licei. Ser. 3, Mem. della Class. di Sci. fis. mat. e at. publisher not identified, 1877.

[CS18] B. Cisneros and C. Segovia. An approximation for the number of subgroups. arXiv:1805.04633, 2018.

[Dem68] P. Dembowski. Finite geometries. Classics in Mathematics 44. SpringerVerlag, 1968.

[Grü09] B. Grünbaum. Configurations of points and lines, volume 103 of Graduate Studies in Mathematics. American Mathematical Society, Providence, RI, 2009.

[HK93] S. Hong and Jin H. Kwak. Regular fourfold coverings with respect to the identity automorphism. J. Graph Theory, 17(5):621-627, 1993.

[HKMP13] A. M. Hinz, S. Klavžar, U. Milutinović, and C. Petr. The tower of Hanoimyths and maths. Birkhäuser/Springer Basel AG, Basel, 2013. With a foreword by Ian Stewart.

[KO16] M. Kosuda and M. Oura. On the centralizer algebras of the primitive unitary reflection group of order 96. Tokyo J. Math., 39(2):469-482, 2016.

[Lev29] F.W. Levi. Geometrische Konfigurationen. Mit einer Einführung in die Kombinatorische Flächentopologie. (S. Hirzel, Leipzig), 1929.

[Li01] P. Li. On the universal embedding of the $\operatorname{Sp}_{2 n}(2)$ dual polar space. J. Combin. Theory Ser. A, 94(1):100-117, 2001.

[McC00] P. McClurg. On the universal embedding of dual polar spaces of type $\operatorname{Sp}_{2 n}(2)$. J. Combin. Theory Ser. A, 90(1):104-122, 2000.

[MR05] N. Moreira and R. Reis. On the density of languages representing finite set partitions. J. Integer Seq., 8(2):Article 05.2.8, 11, 2005.

[MS74] J. W. Milnor and J. D. Stasheff. Characteristic classes. Princeton University Press, Princeton, N. J.; University of Tokyo Press, Tokyo, 1974. Annals of Mathematics Studies, No. 76. 
[PS13] T. Pisanski and B. Servatius. Configurations from a graphical viewpoint. Birkhäuser Advanced Texts. Springer, 2013.

[Ric00] H. W. Richmond. The figure formed from six points in space of four dimensions. Math. Ann., 53(1-2):161-176, 1900.

[RS06] M. Rosas and B. Sagan. Symmetric functions in noncommuting variables. Trans. Amer. Math. Soc., 358(1):215-232, 2006.

[Seg19] C. Segovia. The classifying space of the $1+1$ dimesional $G$-cobordism category. arXiv:1211.2144, 2019.

[Slo] N. J. A. Sloane. The on-line encyclopedia of integer sequences. Electronically. https://oeis.org/.

[SW15] C. Segovia and M. Winklmeier. On the density of certain languages with $p^{2}$ letters. Electron. J. Combin., 22(3):\#P3.16, 2015.

[S+09] SageMath, the Sage Mathematics Software System (Version 8.0), The Sage Developers, 2017, https://www.sagemath.org. 